\title{
Electroweak gauge boson polarisation at the LHC
}

\author{
W.J. Stirling, E. Vryonidou \\ Cavendish Laboratory, J.J. Thomson Avenue, Cambridge CB3 OHE, UK
}

\begin{abstract}
We study the polarisation of gauge bosons produced at the LHC. Polarisation effects for $W$ bosons manifest themselves in the angular distributions of the lepton and in the distributions of lepton transverse momentum and missing transverse energy. The distributions also depend on the selection cuts, with kinematic effects competing with polarisation effects. The polarisation is discussed for a range of different processes producing $W$ bosons: $W+$ jets, $W$ from top (single and pair) production, $W$ pair production and $W$ production in association with a $Z$ or Higgs boson. The relative contributions of the different polarisation states varies from process to process, reflecting the dynamics of the underlying hard-scattering process. We also present results for the polarisation of the $Z$ boson produced in association with QCD jets at the LHC, and comment on the differences between $W$ and $Z$ production.
\end{abstract}




\section{Contents}

1. Introduction 1

2. Polarisation in $W+$ jets production 3

$2.1 \quad W+$ one jet production

2.2 Effect of cuts on the polarisation results

$2.3 W$ plus more jets production 12

3. $W$ bosons from top pair production 13

4. $W$ bosons from other hard-scattering processes 15

5. $Z$ boson polarisation 21

$5.1 Z$ plus jets

$5.2 W$ plus $Z$

6. Conclusions 23

\section{Introduction}

The production of vector bosons has been extensively studied at past and present highenergy collider experiments. Measurements of processes involving weak vector bosons are important both for confirming Standard Model (SM) electroweak predictions and in the search for evidence of New Physics. Recent LHC analyses have studied many different processes involving $W$ and $Z$ bosons. These include $W$ and $Z$ production both in association with QCD jets and in purely electroweak processes.

In the search for New Physics it is important to be able to accurately predict the corresponding SM backgrounds. In this context, $W, Z+$ jets production has been extensively studied in the literature, with NLO corrections recently calculated for up to four associated jets $[1,2]$. Apart from precise knowledge of the SM prediction, another important tool in the search for New Physics is the use of underlying properties to distinguish potential signals from the SM background. One such property is the polarisation of gauge bosons and the resulting distributions of lepton transverse momentum and missing transverse energy. $W$ bosons produced along the beam direction have long been known to be predominantly left-handed at the LHC [3]. In the study of Ref. [4], which considered the lepton transverse momentum and missing energy distributions, it was observed that $W$ bosons produced at the LHC in association with QCD jets are in general preferentially left-handed. The underlying physics leading to this observation has been explored in [5] for $W+1$ jet, where 
the polarisation of $W$ bosons at non-zero transverse momentum, the angular distributions of the final-state decay leptons and the corresponding angular coefficients have been considered in detail. The dependence of the polarisation on the number of jets and the NLO pQCD corrections has also been investigated, with the results shown to be rather stable.

The CMS collaboration at the LHC has measured [6] the polarisation of $W$ bosons produced in association with QCD jets at large transverse momentum, and demonstrated good agreement with the SM predictions presented in [5]. ATLAS has also very recently reported the measurement of the $W$ polarisation using the 2010 LHC data set in [7]. In addition to the polarisation fractions of $W$ bosons, the angular distributions of the produced leptons and the corresponding angular coefficients have also been investigated experimentally. An earlier analysis of the Tevatron $(p \bar{p})$ data has extracted the angular distribution of leptons from $W$ decays [8] and the corresponding angular coefficients. Similarly, the full spin density matrix of the $W$ boson has been investigated in a series of phenomenological studies, see for example [9-12].

Measurements of the polarisation of $W$ bosons from other processes have also been undertaken by high-energy collider experiments. The polarisation of $W$ bosons from top pair production and decay has been investigated in the literature and measured by the Tevatron experiments [13-16]. In this case the polarisation of the positively charged $W$ boson in the top rest frame is found to be predominantly longitudinal. The results are consistent with the SM predictions and therefore measurements of the $W$ polarisation in top pair production have also been employed to set limits on anomalous $W t b$ couplings [17]. The polarisation of weak bosons produced in pairs has been discussed in [18-20] and measurements of the $W$ polarisation have been performed by LEP experiments for $W$ pair production in $[21,22]$. The results were used to set limits on anomalous triple gauge boson couplings in [23].

At the LHC, further exploration of the polarisation of gauge bosons will provide useful insight on their production mechanisms in kinematic regions not previously accessible at other colliders. This will prove useful both as a confirmation of the SM predictions but more importantly in the search for new interactions which can lead to different polarisation behaviour. In the absence of any deviation from the SM, measurements can be used to set more stringent limits on anomalous couplings, similarly to the Tevatron and LEP studies.

In this study we revisit the polarisation of $W$ bosons produced in association with QCD jets in high-energy hadron-hadron collisions: how this is defined and computed and how it affects the shapes of the observable (lepton and missing energy) distributions. In Section 2 we also investigate how the angular distributions of the decay leptons is affected by the introduction of selection cuts on the leptons and jets. In Section 3 we extend the analysis to $W$ bosons from top pair production and decay, and then in Section 4 to a number of other processes in which $W$ bosons are produced. In Section 5 we present corresponding results for the $Z$ boson, commenting on the differences between the two weak bosons, and we present our conclusions in Section 6. 


\section{Polarisation in $W+$ jets production}

\section{$2.1 W+$ one jet production}

The observation that led the authors of [5] to study the polarisation of $W$ bosons at large transverse momentum was the characteristic shape of distributions of the $W$ decay products. The charge asymmetry ratio of $W$ and lepton $p_{T}$ distributions is strongly influenced by polarisation effects both in the $W$ boson production and decay. ${ }^{1}$ In this section we begin by considering $W+1$ jet production to explore the $W$ polarisation effects. The processes involved in $W+1$ jet production provide a simpler underlying mechanism and give a more direct handle on the kinematics. Results for more than one jet will be presented in subsequent sections.

The ratio of distributions for $W+1$ jet production is shown in Fig. 1 for 7 and $14 \mathrm{TeV}$ proton-proton collisions, for a standard set of final-state cuts appropriate for $W$ studies as listed in the figure caption, and with renormalisation and factorisation scales set to $M_{W}$. This result and all subsequent quantitative results in this work have been obtained using LO cross sections and MSTW2008LO PDFs [24]. We will comment on the influence of next-to-leading order (NLO) corrections below.

The asymmetry in the $\mathrm{W}^{ \pm} p_{T}$ distributions is manifest in Fig. 1, with the ratio of $W^{+}$ and $W^{-} p_{T}^{W}$ distributions increasing monotonically with increasing $W$ transverse momentum. This is simply due to valence quarks becoming more important at high subprocess energies (equivalently, large momentum fractions $x$ ). At large $x$ the dominance of the valence $u$-quark parton distribution over that of the $d$-quark leads to an increasing ratio of $W^{+}$over $W^{-}$. The effect is stronger at $7 \mathrm{TeV}$ than at $14 \mathrm{TeV}$, since the corresponding $x$ values are larger for the same $p_{T}^{W}$. This asymmetry between $W^{+}$and $W^{-}$at the LHC can also be used as a diagnostic tool for the presence of New Physics, see [25].

The difference between the electron and positron $p_{T}$ distributions is characteristic of the dominant $W$ left-handed polarisation. In contrast to the $W^{+} / W^{-}$ratio, the ratio of the corresponding lepton transverse momentum distributions decreases from small to medium $p_{T}$. The small increase at very high $p_{T}$ is again related to the relative strength of valence quark PDFs at high $x$. In contrast, the corresponding ratio for the missing transverse energy is an increasing function across the whole $p_{T}$ range. An effect of the same origin is observed in the characteristic shape of the ratio of the charged lepton $p_{T}$ distribution to the missing $E_{T}$ distribution, where the polarisation properties translate into a decreasing ratio for $W^{+}$and an increasing ratio for $W^{-}$, as shown in Fig. 2 for the LHC at $7 \mathrm{TeV}$. As already noted in [5], similar shapes are obtained when more jets are present. We note here that all following plots presented in this paper are obtained for the LHC at $7 \mathrm{TeV}$.

If we relax the cuts so that the only cut is on the jet $p_{T}>30 \mathrm{GeV}$ (as in [5]), then the result at $7 \mathrm{TeV}$ is shown in Fig. 3, $c f$. Fig. 1 in which a full set of cuts have been imposed. Evidently the basic shape characteristics are still present with the introduction of cuts having little impact at large transverse momenta.

\footnotetext{
${ }^{1}$ In this paper we will focus mainly on differences in the distributions of the $W$ and lepton transverse momenta due to polarisation, but effects of the same origin can be observed in the corresponding rapidity distributions.
} 


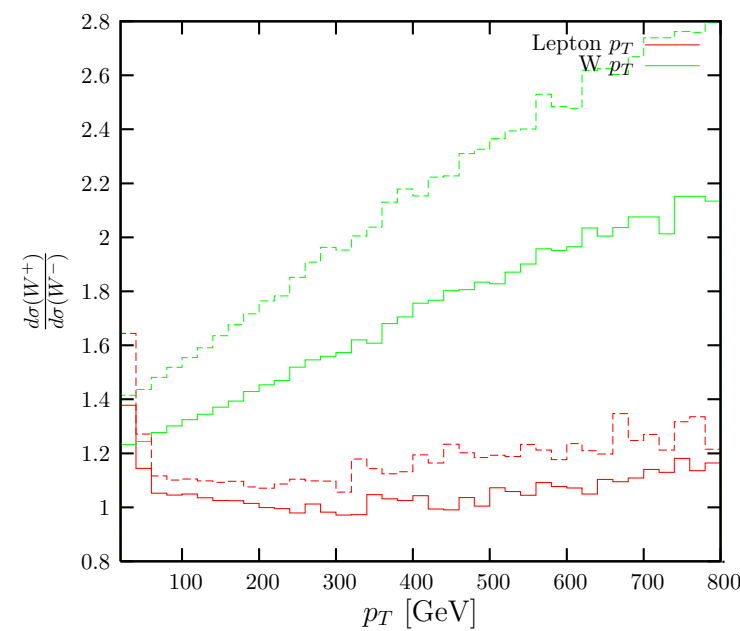

Figure 1: Ratio of differential distributions for $W^{+}$and $W^{-}$for $W+1$ jet. Dashed: $7 \mathrm{TeV}$, solid: $14 \mathrm{TeV}$. Cuts: lepton, jet, missing $p_{T}>20 \mathrm{GeV}$ and $|\eta|<2.5$ for both leptons and jets.

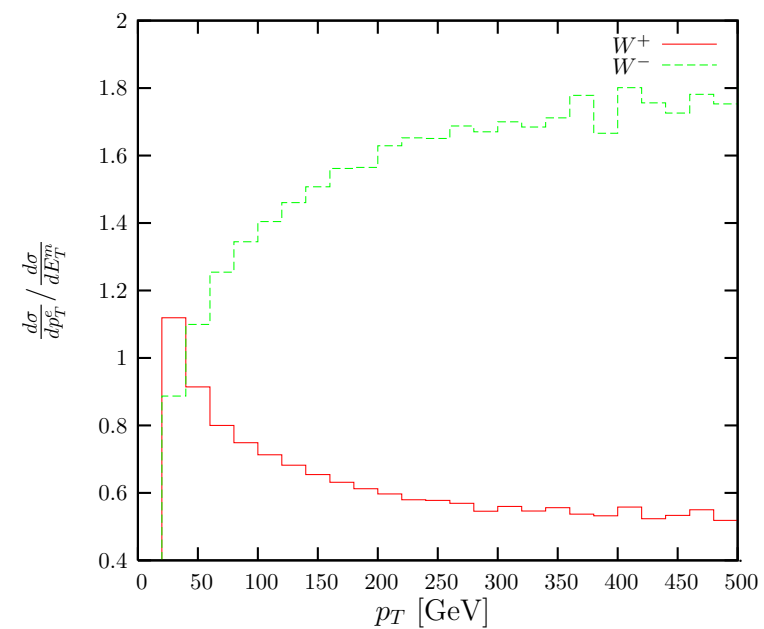

Figure 2: Ratio of the differential distribution of the lepton transverse momentum to the distribution of the missing transverse energy for both $W^{+}$and $W^{-}$at $7 \mathrm{TeV}$. Imposed cuts as in Fig. 1.

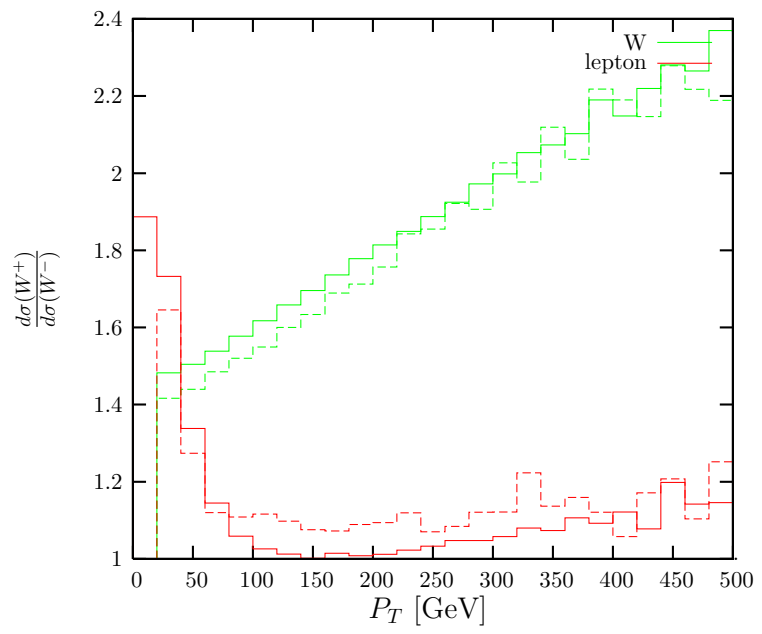

Figure 3: Effect of cuts on the shape of the differential distributions at $7 \mathrm{TeV}$ : only a jet minimum $p_{T}$ cut of $30 \mathrm{GeV}$ (solid) and for the full set of cuts as in Fig. 1 (dashed).

The polarisation fractions of the $W$ boson in the helicity frame corresponding to the distributions shown in Fig. 33 have been obtained in [5]. In general, the angular distribution of the $W^{+}$decay products in the $W^{+}$rest frame is described by:

$$
\frac{1}{\sigma} \frac{d \sigma}{d \cos \theta^{*}}=\frac{3}{8}\left(1-\cos \theta^{*}\right)^{2} f_{L}+\frac{3}{8}\left(1+\cos \theta^{*}\right)^{2} f_{R}+\frac{3}{4} \sin ^{2} \theta^{*} f_{0},
$$

where $\theta^{*}$ is the angle in the $W$ rest frame between the charged lepton and the $W$ flight direction in the lab frame, and $f_{0, L, R}$ are the polarisation fractions. For $W^{-}, f_{R}$ and $f_{L}$ are interchanged. The normalisation is chosen so that $f_{0}+f_{L}+f_{R}=1$ and any dependence 
on the azimuthal angle has been integrated out. In [5] it is noted that $\sigma$ in Eq. (2.1) can be any differential cross section that does not depend on the kinematics of the individual leptons. In the literature the definition of $\theta^{*}$ varies between different studies, with the Collins-Soper frame [9] definition being used extensively.

Based on the structure defined in Eq. (2.1), in order to obtain the polarisation fractions in the helicity frame we use the following expressions (for $W^{+}$):

$$
\begin{aligned}
f_{0} & =2-5\left\langle\cos \theta^{* 2}\right\rangle \\
f_{L} & =-\frac{1}{2}-\left\langle\cos \theta^{*}\right\rangle+\frac{5}{2}\left\langle\cos \theta^{* 2}\right\rangle, \\
f_{R} & =-\frac{1}{2}+\left\langle\cos \theta^{*}\right\rangle+\frac{5}{2}\left\langle\cos \theta^{* 2}\right\rangle .
\end{aligned}
$$

These functions of $\theta^{*}$ can be used on an event-by-event basis as projections to extract the polarisation fractions for any $\sigma$ that does not depend on the kinematics of the individual leptons. In other words, the method assumes full acceptance for the leptons.

The polarisation fractions for $W$ bosons as a function of the $W$ boson transverse momentum have already been obtained in [5] for $W+1$ jet with a simple jet $p_{T}$ cut of $30 \mathrm{GeV}$. We reproduce these results (for the LHC at $7 \mathrm{TeV}$ ) for both $W^{+}$and $W^{-}$in Fig. 4 where we also show the results as a function of the $W$ rapidity. The small difference between the $W^{+}$and $W^{-}$polarisations is due to the difference between the valence $u-$ and $d$-quark PDFs that forces the $W^{+}$to be slightly more left-handed. We also see the longitudinal fraction falling to zero at large transverse momentum, in agreement with the equivalence theorem $[26]$.

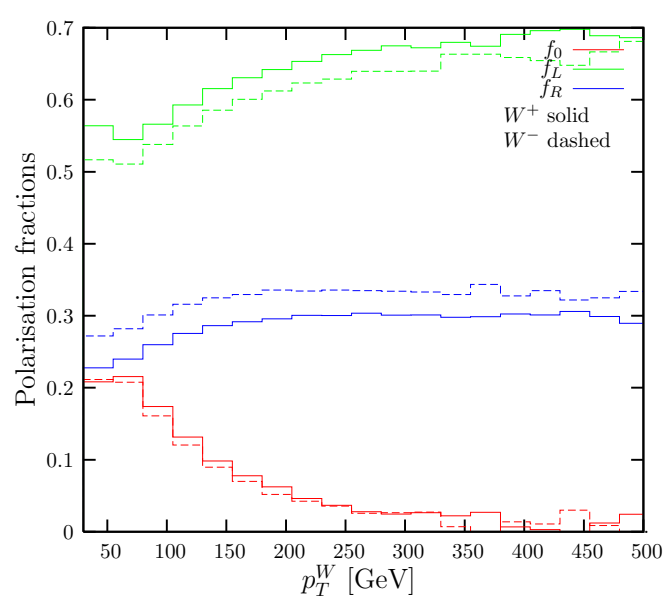

(a)

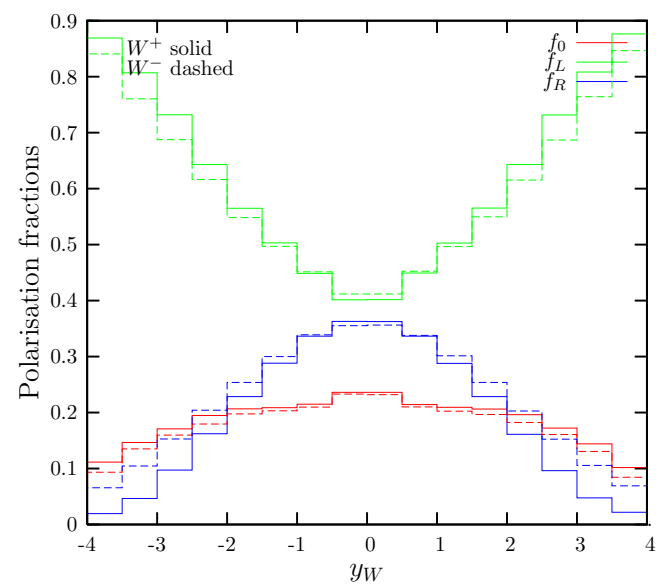

(b)

Figure 4: Polarisation fractions as a function of a) $p_{T}^{W}$ and b) $y_{W}$ for $7 \mathrm{TeV}$ and a jet $p_{T}$ $\left(=p_{T}^{W}\right.$ for $W+1$ jet $)$ cut of $30 \mathrm{GeV}$ for both $W^{+}$and $W^{-}$.

In the case of $W+1$ jet production the cut on the jet transverse momentum effectively acts as a cut on the $W p_{T}$, which explains the cut-off at low $p_{T}^{W}$. By decreasing the jet $p_{T}$ 
cut we obtain the limiting fractions for $p_{T}^{W} \sim 0$ shown in Fig. 5 . $^{2}$ These are the same as for leading-order $W$ boson production along the beam direction. In this case the polarisation of the $W$ is simply determined by the momentum of the colliding quark and antiquark. The $W$ boson is left-handed if the quark has more momentum than the antiquark, and right-handed otherwise. Longitudinal polarisation is not permitted by angular momentum conservation. The exact values of the fractions at zero transverse momentum therefore depend on the relative values of the corresponding quark and antiquark PDFs. At the LHC, quarks have on average more momentum than antiquarks which explains the difference in values $f_{L}=0.73$ and $f_{R}=0.27$ for $W^{+}$, while the same arguments apply for $W^{-}$leading to $f_{L}=0.68$ and $f_{R}=0.32$. At the Tevatron, quarks and antiquarks have on average the same momentum and therefore we expect the fractions to be closer to 0.5 for $W^{+}$. The values are indeed calculated to be $f_{L}=0.60$ and $f_{R}=0.40$. These are not exactly 0.5 , as the $W^{+}$is primarily produced from a proton $u$-quark and an antiproton $\bar{d}$, with the $u$-quark carrying more momentum on average. In this case the fractions for $W^{-}$are exactly reversed, with $W^{-}$being preferentially right-handed.

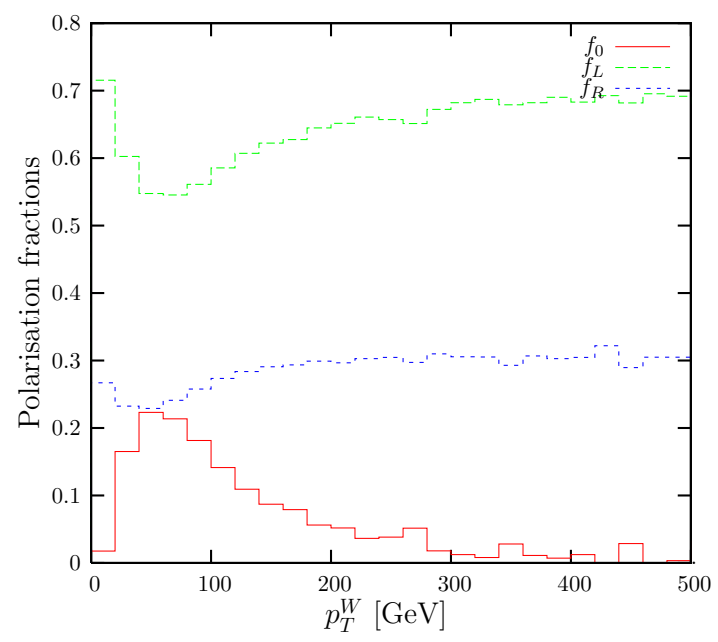

Figure 5: Polarisation fractions as a function of $p_{T}^{W}$ for $7 \mathrm{TeV}$ and a nominal jet/parton $p_{T}$ cut of $0.1 \mathrm{GeV}$ for $W^{+}$.

For $W+1$ jet production, different subprocesses contribute to the total cross section. The analysis for $W+1$ jet production in [5] explains why given the dominant parton subprocesses one expects $W$ bosons to remain predominantly left-handed at high transverse momentum. The argument is based on the spin-1 nature of $W$ and its coupling to lefthanded fermions at the helicity amplitude level.

In addition to the polarisation fractions, which are the diagonal elements of the $W$ boson spin-density matrix, we can also compute the full set of (polar and azimuthal) angular coefficients as a function of the $W$ transverse momentum as in [5] for the LHC

\footnotetext{
${ }^{2}$ In practice we need to impose a small $p_{T}$ cut, as the cross section for $W+1$ jet production is formally divergent at zero $p_{T}$, even though the polarisation fractions are finite in this limit.
} 
and [12] for the Tevatron. The differential cross section is written as:

$$
\begin{aligned}
\frac{1}{\sigma} \frac{d \sigma}{d \cos \theta^{*} d \phi^{*}} & =\frac{3}{16 \pi}\left[\left(1+\cos ^{2} \theta^{*}\right)+A_{0} \frac{1}{2}\left(1-3 \cos ^{2} \theta^{*}\right)+A_{1} \sin 2 \theta^{*} \cos \phi^{*}\right. \\
& \left.+A_{2} \frac{1}{2} \sin ^{2} \theta^{*} \cos 2 \phi^{*}+A_{3} \sin \theta^{*} \cos \phi^{*}+A_{4} \cos \theta^{*}\right],
\end{aligned}
$$

with the angle $\phi^{*}$ defined as in [5]. Integrating over $\phi^{*}$ from 0 to $2 \pi$ we recover Eq. (2.1) and we can relate $f_{0, R, L}$ to the angular coefficients $A_{i}$. Three more coefficients not shown in Eq. (2.5) vanish at LO because of parity invariance. The remainder of the coefficients can be determined using appropriate projections as for the polarisation fractions. We show the LO results obtained for $W+1$ jet for $W^{-}$and $W^{+}$in Fig. 6. At LO $A_{0}=A_{2}$ [10] and therefore we only show $A_{2}$ in the plots. The results are in good agreement with the NLO results of [5] which also include events with more jets.
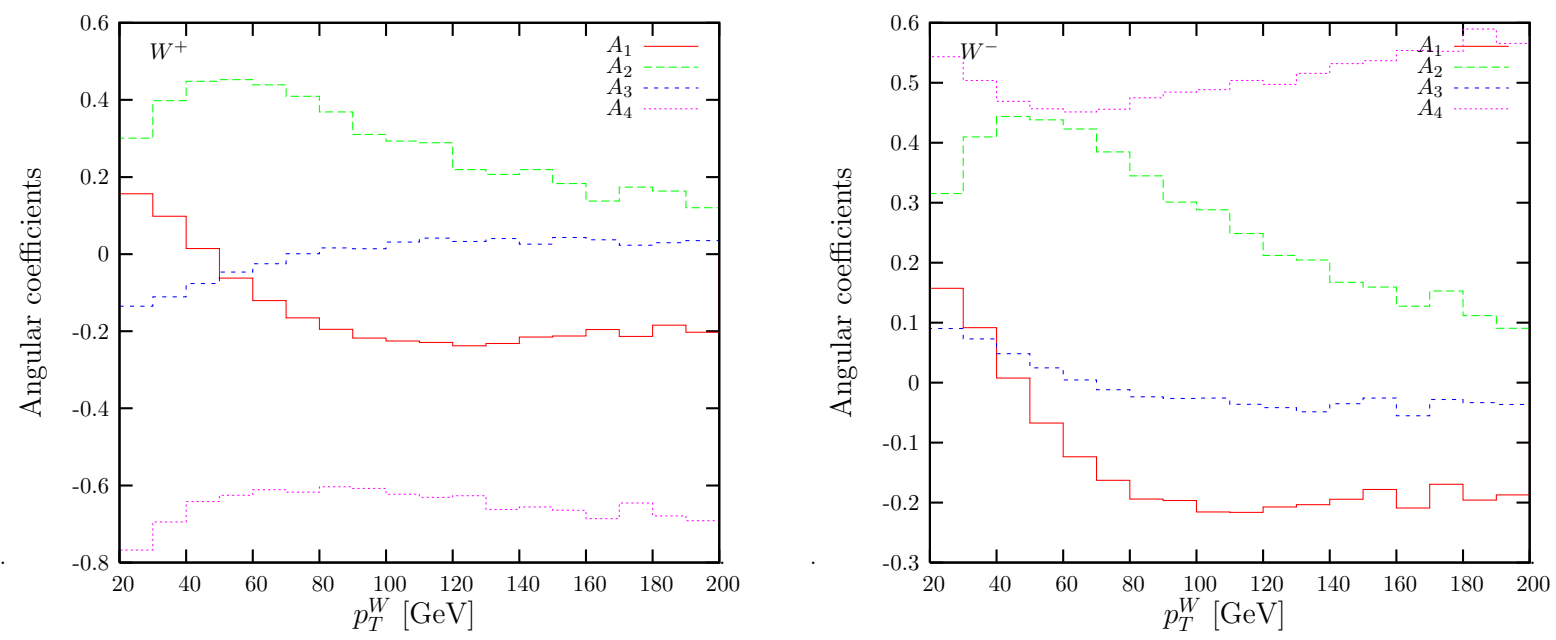

Figure 6: Angular coefficients for $W^{-}$and $W^{+}$plus one jet with no imposed cuts in proton-proton collisions at $7 \mathrm{TeV}$.

The above results were obtained using our own $W+$ jets LO programme. We have checked that our results agree with a calculation using MCFM [27], after matching the electroweak parameters, PDFs and scale choice.

\subsection{Effect of cuts on the polarisation results}

For a more realistic analysis one needs to introduce additional acceptance cuts on the finalstate lepton transverse momenta and rapidities. Indeed such cuts will also serve to reduce backgrounds in searches for New Physics. The impact of cuts has been first noted in [12], where the angular distribution of the weak boson decay products was studied including the effect of introducing kinematic cuts and taking into account the detector energy resolution. In this section we investigate the effect of selection cuts on the angular distributions. Cuts are introduced in turn to disentangle the effect of different cuts. The cuts introduced are: $p_{T}^{j}>20 \mathrm{GeV}, p_{T}^{\ell}>20 \mathrm{GeV}, E_{T}^{m}>20 \mathrm{GeV}$ and $\left|\eta_{\ell, j}\right|<2.5$. The rapidity cut is imposed on both leptons and jets. These are designed to mimick a 'typical' set of cuts employed 
by the experimental collaborations to select $W$ boson events. The first distribution we consider is the charged lepton angular distribution $d \sigma / d \cos \theta^{*}$. The comparison between

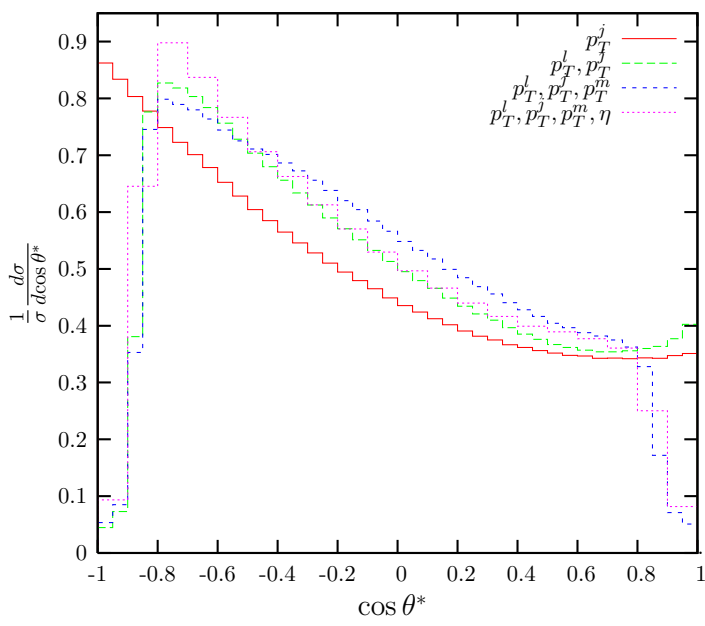

Figure 7: Normalised angular distribution for a set of different selection cuts imposed on final-state leptons and jets for $W^{+}+1$ jet production at $7 \mathrm{TeV}$.

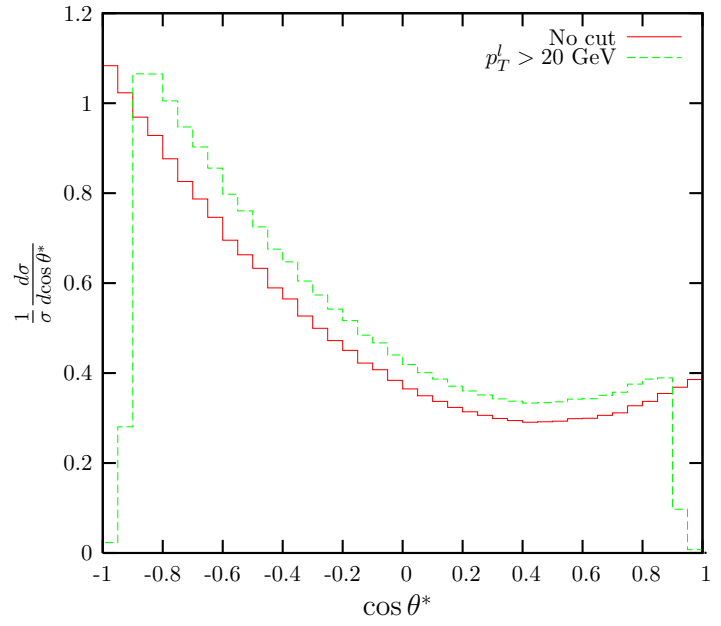

Figure 8: Angular distribution for $\mathrm{LO} W^{+}$ production along the beam direction with no imposed cuts and with a cut on the charged lepton $p_{T}$.

the normalised angular distributions for $W^{+}+1$ jet for different selection cuts is shown in Fig. 7. The cross section gets progressively smaller on the introduction of additional cuts but as we are considering the normalised distributions we only observe the changes in shape.

We note that for $W^{+}+1$ jet production introducing a cut on the lepton transverse momentum reduces the cross section mainly in the region of $\theta^{*} \sim \pi$ and similarly the effect of a cut on the missing transverse energy is more important in the $\theta^{*} \sim 0$ region, as for a left-handed $W^{+}$the neutrino is preferentially emitted in the direction of the $W$. For $\mathrm{LO} W^{+}$production along the beam direction with no associated jet the same effect is seen in the distribution as shown in Fig. 8. However in this case the transverse lepton momentum is exactly balanced by the missing transverse energy. Therefore the cut on the lepton transverse momentum implies the same cut on the missing transverse energy and the normalised angular distribution is modified at both ends. The $W$ rest frame is in this case identified as the centre-of-mass frame and it is therefore clear that imposing a cut on $p_{T}^{\ell}$ suppresses forward or backward scattering.

One can also study the differential distribution in the azimuthal angle $\phi^{*}$ as defined in [5]. The distribution is even in $\phi^{*}$ and therefore the distributions shown in Fig. 9 correspond to $d \sigma / d\left|\phi^{*}\right|$. Again we see that the acceptance cuts do not modify the cross section uniformly over the range of the angle $\phi^{*}$ and accordingly change the shape of the normalised distribution.

In general, we see that the angular distributions change rapidly on the introduction of additional cuts. Regarding the polarisation fractions, we note that no straightforward expression like that in Eq. (2.1) applies once lepton cuts have been introduced, i.e. Eq. (2.1) 


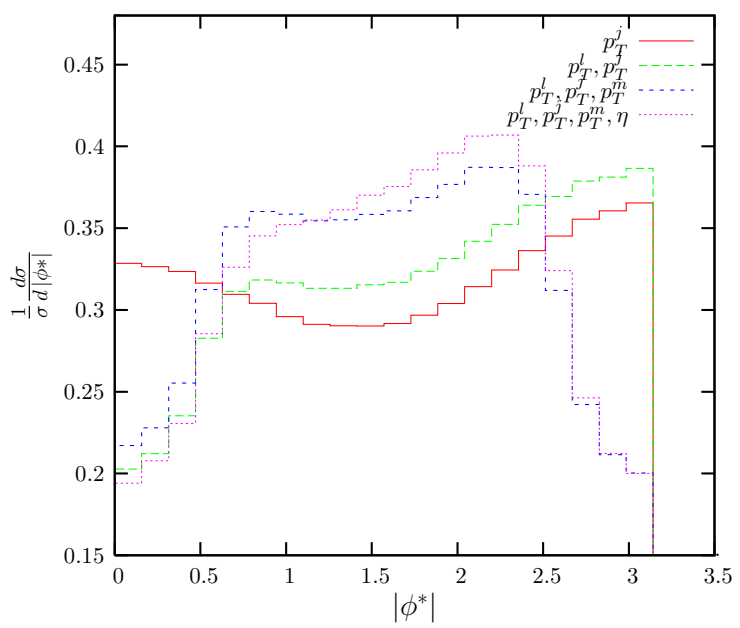

Figure 9: Normalised azimuthal angle distributions for a set of different selection cuts imposed on final-state leptons and jets for $W^{+}+1$ jet production at $7 \mathrm{TeV}$.

is valid only for full $(4 \pi)$ lepton acceptance. One could still use the expressions for the fractions as observables, but these will no longer represent the polarisation fractions. Based on Fig. 7, if we insist on using the same $\theta^{*}$-dependent projections we expect them to give significantly different results. As an example, we show in Fig. 10 the result obtained for a lepton transverse momentum cut of $20 \mathrm{GeV}$, in addition to the jet transverse momentum cut of $20 \mathrm{GeV}$, for $W^{+}+1$ jet production at $7 \mathrm{TeV}$.

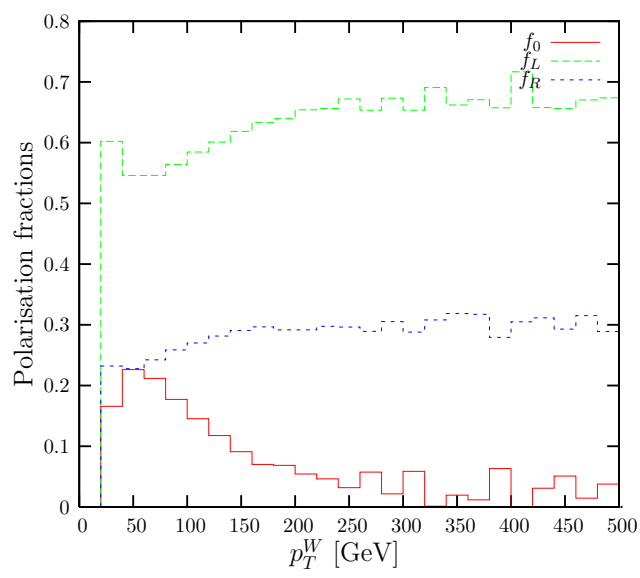

(a)

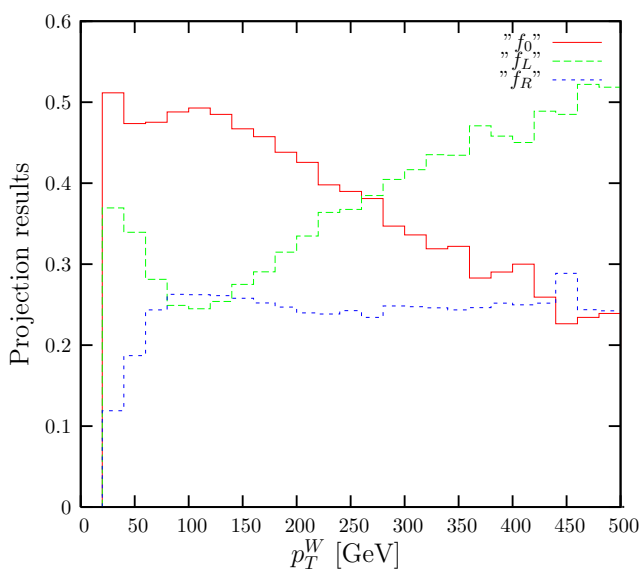

(b)

Figure 10: Projection results as a function of $p_{T}^{W}$ for $7 \mathrm{TeV}$ for $W^{+}+1$ jet with a jet $p_{T}$ cut of $20 \mathrm{GeV}$ and a) without and b) with a cut for charged leptons $p_{T}^{l}>20 \mathrm{GeV}$.

In Fig. 10 we note the rather dramatic impact of the lepton cuts on the results. Contrary to what one might naively expect, even a modest lepton $p_{T}$ cut of $20 \mathrm{GeV}$ modifies the results for $p_{T}^{W}$ up to about $500 \mathrm{GeV}$. This can be explained by the fact that $W$ bosons are predominantly left-handed at high $p_{T}^{W}$, creating an asymmetry in the distributions of lepton transverse momentum and missing transverse energy. The distributions of lepton 
transverse momentum and missing transverse energy are shown in Fig. 11 for a cut of $400 \mathrm{GeV}$ on $p_{T}^{W}$. Since the cross section is a rapidly falling function of $p_{T}^{W}$ most of the contribution comes from $W$ bosons with $p_{T}$ just above $400 \mathrm{GeV}$. The two distributions are peaked at opposite ends, with the neutrino along the $W^{+}$direction most of the time as the $W^{+}$is predominantly left-handed. For predominantly right-handed $W^{+}$we expect the peaks to switch positions and for a purely longitudinal $W^{+}$we expect the same shape for both and a peak at around half the $p_{T}^{W}$ cut value. Similar arguments apply for $W^{-}$, but in this case the distributions are interchanged. We note that the shape of the lepton $p_{T}$ resembles the angular distribution $d \sigma / d \cos \theta^{*}$. This explains why the effect of a modest lepton $p_{T}$ cut extends to the region of very high $p_{T}^{W}$.

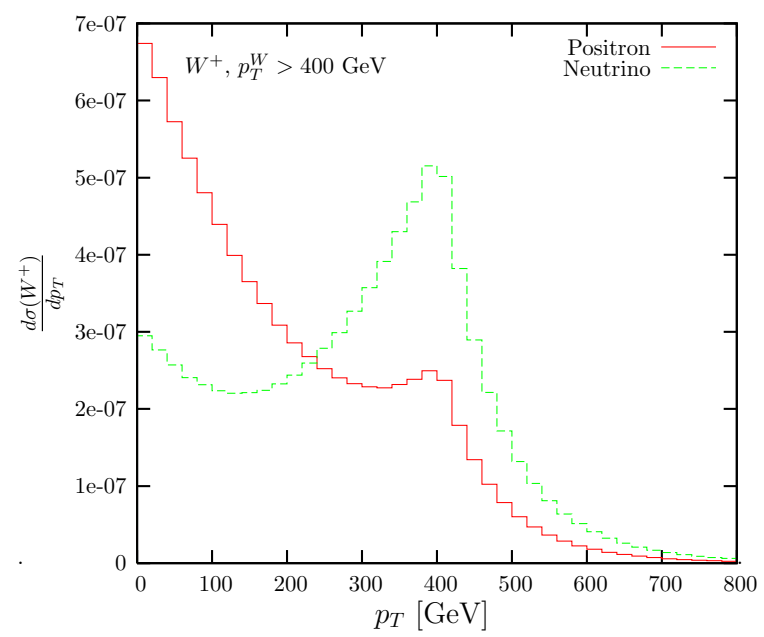

Figure 11: Lepton $p_{T}$ and missing transverse energy distributions for a high $p_{T}^{W}$ cut for $W^{+}+1$ jet production.

As already discussed above, the definition of the polarisation fractions was based on full acceptance in the azimuthal angle $\phi^{*}$ and in general no constraint on the kinematics of the individual leptons. Introducing lepton cuts leads to a dependence of the fractions on other components of the $W$ spin density matrix. Experimentally, lepton cuts are imposed to accommodate finite detector acceptance. In an analysis aimed at extracting the $W$ polarisation, experiments must correct for their lepton cuts before using the projections to obtain the polarisation fractions.

In principle we could still use the expressions for the projections as observables even when lepton cuts have been applied. In this case in order to explain the impact of cuts we need to study the dependence on the observables on which we impose the cuts, e.g. the lepton rapidity and the lepton transverse momentum. The results for the lepton transverse momentum and rapidity are shown in Fig. 12. We see in these plots that " $f_{0}$ " " $f_{L}$ " and " $f_{R}$ " are rapidly changing functions of the two observables which is reflected in the severe impact of the cuts on the results. We note here that once we consider the projections as a function of other variables which depend on the individual kinematics of the leptons we observe that these can become negative. This is not surprising, as these expressions no longer represent the polarisation fractions. 

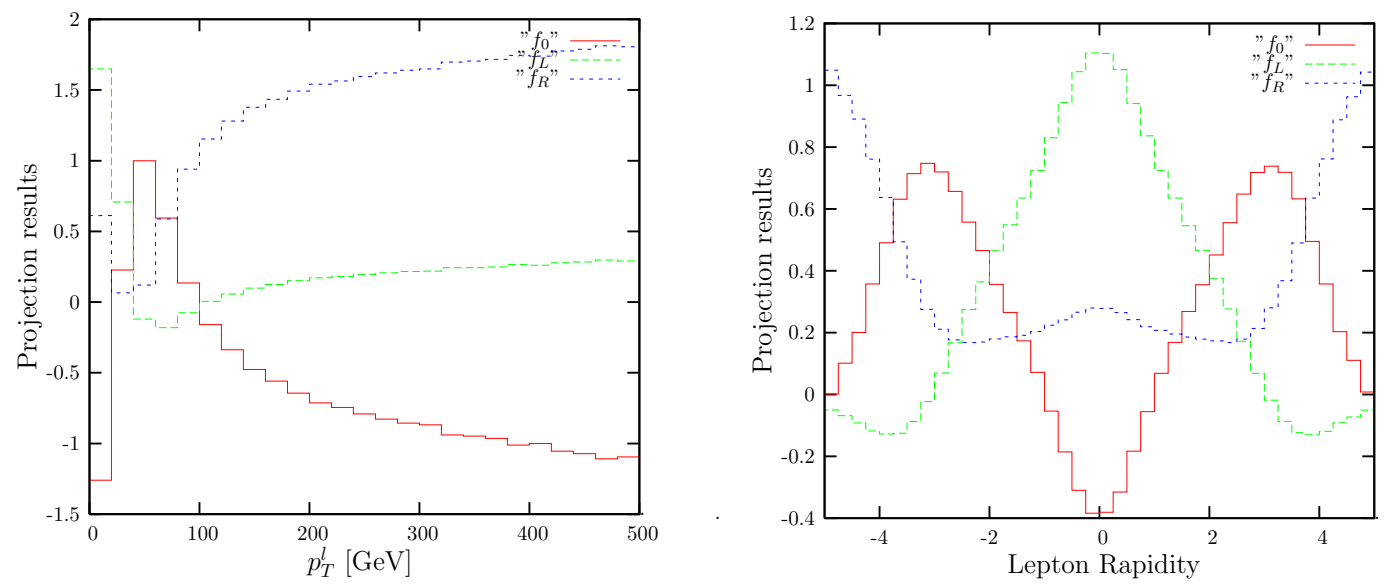

Figure 12: Projection results for $W^{+}$as a function of the lepton $p_{T}$ and rapidity with $p_{T}^{j}>20 \mathrm{GeV}$.

\begin{tabular}{|c|c|c|c|}
\hline Cuts & "f $f_{0}$ & " $f_{L} "$ & " $f_{R} "$ \\
\hline$p_{T}^{j}>30 \mathrm{GeV}$ & 0.20 & 0.56 & 0.23 \\
\hline$p_{T}^{j}>20 \mathrm{GeV}$ & 0.18 & 0.59 & 0.23 \\
\hline$p_{T}^{j}>20 \mathrm{GeV}, p_{T}^{l}>20 \mathrm{GeV}$ & 0.50 & 0.35 & 0.15 \\
\hline$p_{T}^{j}>20 \mathrm{GeV}, p_{T}^{l}>20 \mathrm{GeV}, p_{T}^{m}>20 \mathrm{GeV}$ & 0.68 & 0.29 & 0.03 \\
\hline$p_{T}^{j}>20 \mathrm{GeV}, p_{T}^{l}>20 \mathrm{GeV}, p_{T}^{m}>20 \mathrm{GeV},\left|\eta_{l, j}\right|<2.5$ & 0.59 & 0.36 & 0.05 \\
\hline
\end{tabular}

Table 1: Comparison of results for the different cuts. The values represent polarisation fractions only for the first two rows.

The results for the expressions (not the polarisation fractions except for rows 1 and 2) for different cuts are given in Table 1 .

The above predictions are obtained using a Monte Carlo event generator programme from which we know exactly the momentum of the $W$ boson. Of course experimentally it is impossible to reconstruct exactly and unambiguously the momentum of the $W$ boson, as one can only measure the transverse momentum of the neutrino, unless a $W$ mass contraint is applied on the neutrino-electron pair (see for example the Tevatron polarisation studies in top pair production in [14]). Even when the extra mass constraints are applied, an ambiguity remains and a further selection needs to be made. Therefore a straightforward extraction and use of the angle $\theta^{*}$ to define the polarisation fractions is not feasible. The first measurement of the polarisation of the $W$ boson by CMS [6] introduced the variable $L_{p}$, defined as:

$$
L_{p}=\frac{\vec{p}_{T}(l) \cdot \vec{p}_{T}(W)}{\left|\vec{p}_{T}(W)\right|^{2}}
$$

where all quantities can in principle be reconstructed. In the limit of very high $W$ transverse momentum $\cos \theta^{*}=2\left(L_{p}-1 / 2\right)$. For purposes of comparison with CMS, we also calculate the distribution of $L_{p}$, applying the cuts given in [6] for the muon channel. The cut 
imposed on $p_{T}^{W}$ is $50 \mathrm{GeV}$. We note that to approach as closely as possible the CMS results we prefer the muon channel, as it is less affected by backgrounds (as shown in [6]). We note that these are parton-level results, with no detector simulation obtained for $W+1$ jet. The experimental results include events with more jets, as there is no specific jet number requirement. The normalised distributions for $W^{-}$and $W^{+}$are shown in Fig. 13. The shape of the $L_{p}$ distributions agrees reasonably well with the results of Fig. 2 in the CMS paper. In the plot we also show the distribution of $L_{p}^{h}=\left(\cos \theta^{*}+1\right) / 2$ to investigate the possibility of using $L_{p}$ to recover $\cos \theta^{*}$. The CMS analysis imposes a cut of $50 \mathrm{GeV}$ on the $W$ transverse momentum but the two distributions differ significantly, with the experimental distribution extending above one and below zero. Agreement improves for a $p_{T}^{W}$ cut of $100 \mathrm{GeV}$, as also shown in Fig. $13 \mathrm{~b}$ ). The results for the polarisation fractions in [6] are obtained using the template method to extract the distributions of $L_{p}$ for pure longitudinal, left- and right-handed samples and then fitted to the data to obtain the polarisation fractions. This accounts both for the effect of the selection cuts and the mismatch of $L_{p}$ and $L_{p}^{h}$, as shown in Fig. 13 .

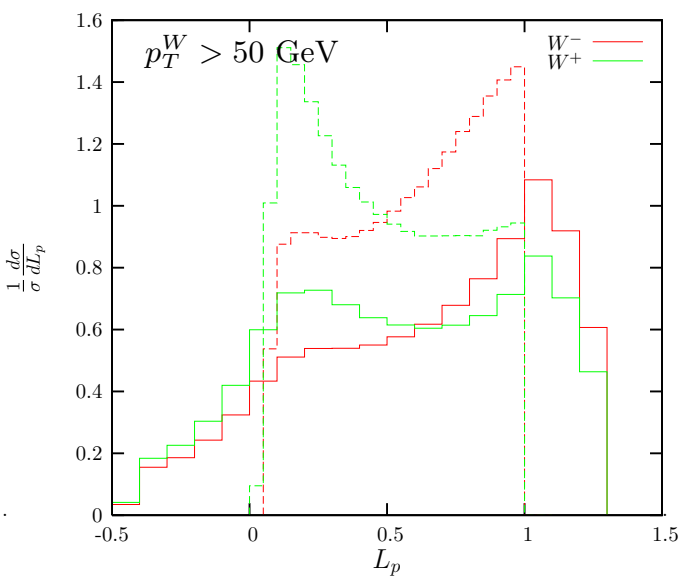

(a)

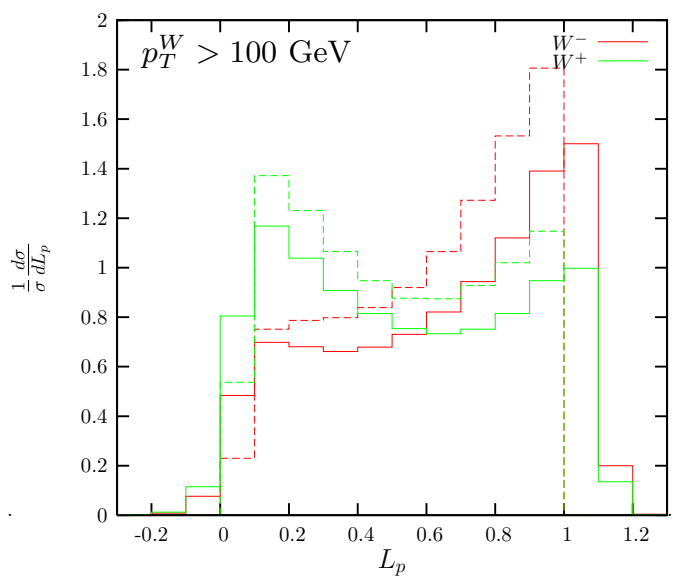

(b)

Figure 13: Normalised distributions for the variable $L_{p}$ for $W^{-}$and $W^{+}$with the CMS cuts. $P_{W}^{T}$ cut increased from a) 50 to b) $100 \mathrm{GeV}$. Solid lines show $L_{p}$ as defined above while dashed lines show $L_{p}^{h}$.

\section{$2.3 W$ plus more jets production}

The results of the previous section have been obtained by considering $W+1$ jet production. Similar considerations can be made for $W+n$ jets with $n \geq 2$. The $W+n$ jets cross section falls with increasing number of jets, as each additional jet is associated with an extra power of $\alpha_{S}$. At the $7 \mathrm{TeV}$ LHC, with a cut of $30 \mathrm{GeV}$ on the transverse momentum of all jets, we obtain the following LO results for $\sigma\left(W^{+}+n\right.$ jets) for the electron decay channel (with no cuts on the leptons or missing energy): $0.611 \mathrm{nb}$ for one jet, $0.215 \mathrm{nb}$ for 2 jets and $0.0741 \mathrm{nb}$ for 3 jets. These results are obtained by running separately the $W+1,2$ and 3 jets routines and checking that all produced jets pass the $p_{T}$ cut. In practice the experimental 
$W+1$ jet sample will contain events in which two jets were emitted but one of them falls outside the acceptance region of the detector etc. Effective use of MC generators is needed to correctly account and correct for this effect.

Similarly to the $W+1$ jet analysis, we study the lepton angular distribution in the $W$ rest frame as shown in Fig. 14 for $W^{+}+2$ jets for a cut of $30 \mathrm{GeV}$ on $p_{T}^{j}$. We note that the shape is identical to that for $W^{+}+1$ jet. The polarisation fractions are calculated using the same method as for $W+1$ jet. An additional cut that needs to be set for two or more jets is a cut on the separation of two jets in order for them to be considered distinct jets. The cone separation variable $R$ has a negligible effect on the polarisation fractions. Moreover we have checked using MCFM that NLO corrections have no sizable impact on the polarisation fractions. The definition of the polarisation fractions as ratios over the total cross sections helps reduce the sensitivity to the NLO corrections. In [5] it has been shown using SHERPA [28] that the results remain stable even when parton shower effects are taken into account.

For comparison we collect in Fig. 15 the results of the polarisation fractions for 1, 2 and 3 jets with a jet transverse momentum cut of $30 \mathrm{GeV}$. As already noted in [5], even though the kinematics become more complicated with an increasing number of jets the polarisation fractions are not very sensitive to the number of jets, with changes only observed at low $W$ transverse momentum and $W$ bosons remaining predominantly left-handed at large transverse momentum.

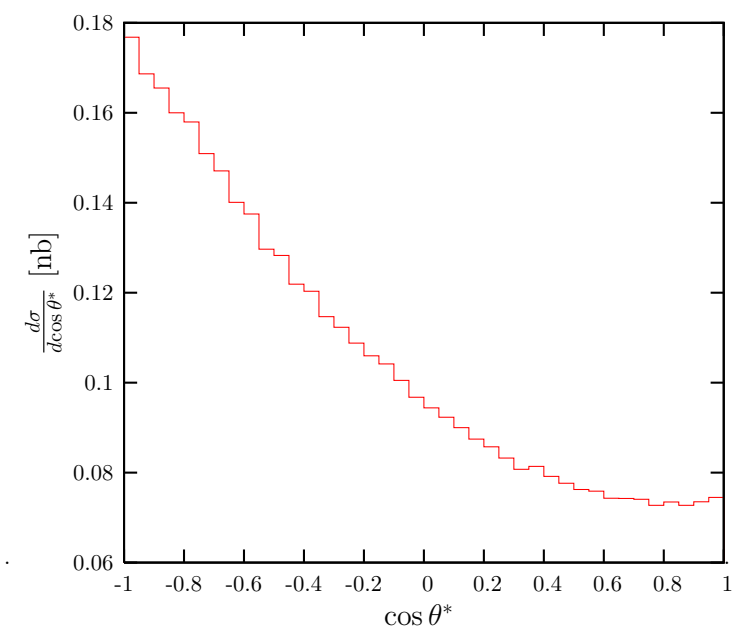

Figure 14: Angular distribution $d \sigma / d \cos \theta^{*}$ for $W^{+}+2$ jets with $p_{T}^{j}>30 \mathrm{GeV}$ at $7 \mathrm{TeV}$.

\section{3. $W$ bosons from top pair production}

In addition to the production of $W$ bosons in association with QCD jets, the polarisation properties of $W$ bosons from other sources can also be investigated. The polarisation of $W$ bosons from top pair production has been measured at the Tevatron both by CDF $[15,16]$ and D0 [13,14]. The projections used are defined in [17], where the polarisation of $W$ bosons from top decays has been used as a probe of anomalous $W t b$ couplings. The projections 


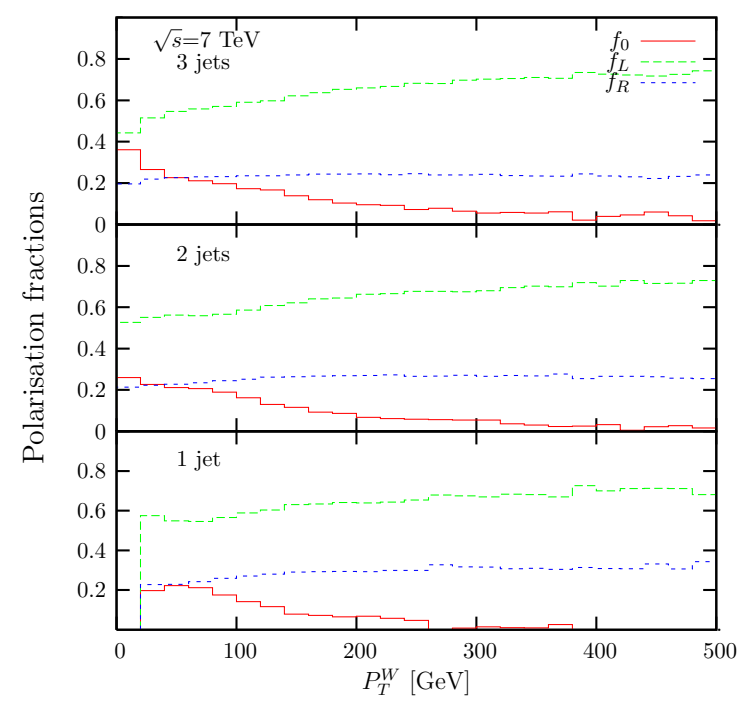

Figure 15: Polarisation fractions for $W^{+}+1,2$ and 3 jets with $p_{T}^{j}>30 \mathrm{GeV}$ at $7 \mathrm{TeV}$ obtained using MCFM.

differ from those used for $W+$ jets, as the angle $\theta^{*}$ is defined in the $W$ rest frame relative to the $W$ direction in the top rest frame.

We begin by employing the projections of [17] to reproduce the overall SM polarisation fractions of $W$ bosons from top pair production given in [17] and measured at the Tevatron. The overall results show that for $W^{+}$bosons from top pair production $f_{0}=0.70$ and $f_{L}=0.30$. The results can also be extracted from analytic expressions involving the $W$ and top masses (for massless $b$-quarks):

$$
f_{0}=\frac{m_{t}^{2}}{m_{t}^{2}+2 m_{W}^{2}} \quad \text { and } \quad f_{L}=\frac{2 m_{W}^{2}}{m_{t}^{2}+2 m_{W}^{2}} .
$$

The fractions obtained from the Monte Carlo simulation are shown as a function of the $W$ transverse momentum in Fig. 16, where no acceptance cuts have been imposed. For $W^{-}$the result is $f_{0}=0.70$ and $f_{R}=0.30$. In contrast to $W+$ jets production we notice that the polarisation fractions are constant and there is essentially no dependence of the polarisation fractions on the $W p_{T}$. This follows naturally from the way polarisation is defined using the top rest frame.

Unlike in the case of $W+$ jets production, in this process $W^{+}$and $W^{-}$are exactly equivalent as these are always produced in pairs from the decaying top-anti-top pair, hence the polarisation fractions are related by $f_{0}^{+}=f_{0}^{-}$and $f_{R}^{+}=f_{L}^{-}$. As for $W+$ jets production we are interested in identifying how the polarisation of the $W$ transforms into an asymmetry in the charged lepton and neutrino kinematics visible in the shape of the distributions. We show the ratio of the $W^{+}$lepton transverse momentum and missing energy distributions in Fig. 17. Considering the symmetric production mechanism this ratio satisfies

$$
\frac{d \sigma(t \bar{t}) / d p_{T}^{e^{+}}}{d \sigma(t \bar{t}) / d p_{T}^{m}}=\frac{d \sigma(t \bar{t}) / d p_{T}^{e^{-}}}{d \sigma(t \bar{t}) / d p_{T}^{m}}
$$


The ratio of the charged lepton $p_{T}$ and missing transverse energy distributions obtained for events where the $W^{+}$decays leptonically and the $W^{-}$hadronically is identical to that where the $W^{-}$decays leptonically and the $W^{+}$hadronically. Here we assume that only one of the two $W$ bosons decays leptonically so that there is only one charged lepton produced in the event. We note that the shape of the ratio of the distributions is similar to that of $W^{+}$in Fig. 2. This is expected as the asymmetry between the lepton transverse momentum and the missing transverse energy originates in the difference between the two transverse polarisation fractions. In both cases the produced $W^{+}$is preferentially left-handed, which explains the decrease of the ratio with increasing $p_{T}$. In contrast, the produced $W^{-}$is preferentially right-handed, leading to an identical ratio of distributions as for $W^{+}$decays from top but fundamentally different from that of $W^{-}+$jets production.

We note that in this case no asymmetry is expected between the number of produced electrons and positrons. Top pair production with one leptonic and one hadronic decay with no other mass selection cuts can be regarded as a background to QCD $W+4$ jets production (or vice versa). A comparison of the lepton and missing energy distributions in

$W+$ jets and $t \bar{t}$ production has been made in [29] where a density plot is used to show the difference between the two processes. The density plot is made using the $W+3$ jets and $W$ from top pair production with just three identified jets. Here we attempt to extract similar density plots using our $W+1$ jet and top pair production programs with no specific requirement on the number of jets. For $W+$ jets the characteristic shape is expected to persist when changing the number of jets as this is determined by the polarisation properties which are found to be stable. The ratio of the double differential cross section in lepton $p_{T}$ and missing transverse momentum $\left(d^{2} \sigma / d p_{T}^{l} d p_{T}^{m}\right)$ of $W$ from top decays to that of QCD $W+$ jets is shown in the density plots of Fig. 18. The same conclusions as in [29] can be drawn, even though we note some differences at low $p_{T}$ which are most likely related to the different number of jets in the process and the choice of selection cuts. In the density plots we note the asymmetry between $W^{-}$and $W^{+}$as the missing $E_{T}$ and $p_{T}^{l}$ space is populated differently by $W+$ jets and $t \rightarrow W$ events. We also note that the most noticeable difference occurs for $W^{-}$as the polarisation changes from left-handed in $W+$ jets to right-handed in top pair production.

In the extraction of polarisation fractions in order to be consistent with our analysis for $W+$ jets we should of course use the same projections with $\theta^{*}$ defined relative to the $W$ direction in the lab frame. The comparison between the two definitions is shown in Fig. 19, together with the distribution for the $W p_{T}$. For $W^{-} f_{R} \Leftrightarrow f_{L}$. It is clear from the plot that the polarisation fractions are highly sensitive to the definition of the angle and therefore frame dependent. The total polarisation fractions obtained by integrating over the whole phase space with no imposed cuts are different. Moreover, use of the lab frame in the definition also introduces a dependence of the fractions on the $p_{T}$ of the $W$ boson.

\section{4. $W$ bosons from other hard-scattering processes}

Apart from QCD $W+$ jets and top pair production, other processes producing $W$ bosons include: single top production, $W^{+} W^{-}$and $W Z$ gauge boson pair production, $W$ plus 


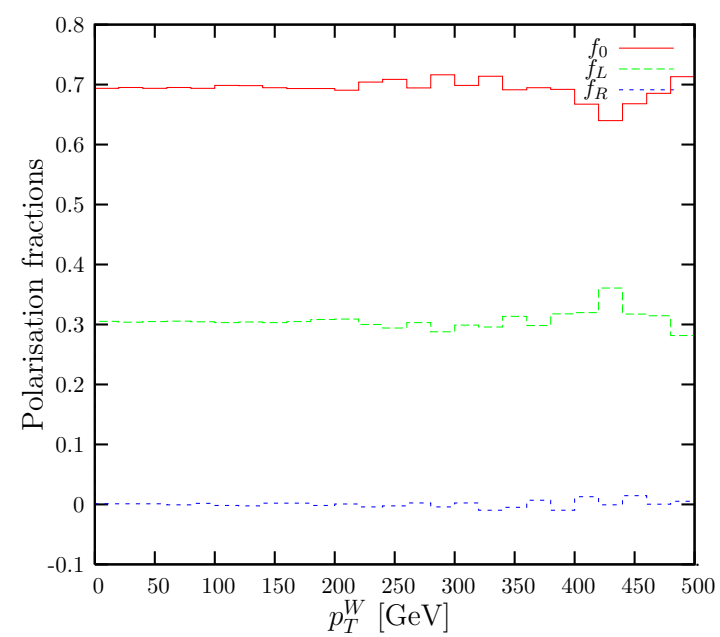

Figure 16: Polarisation fractions for $W^{+}$from top decays with no imposed cuts.

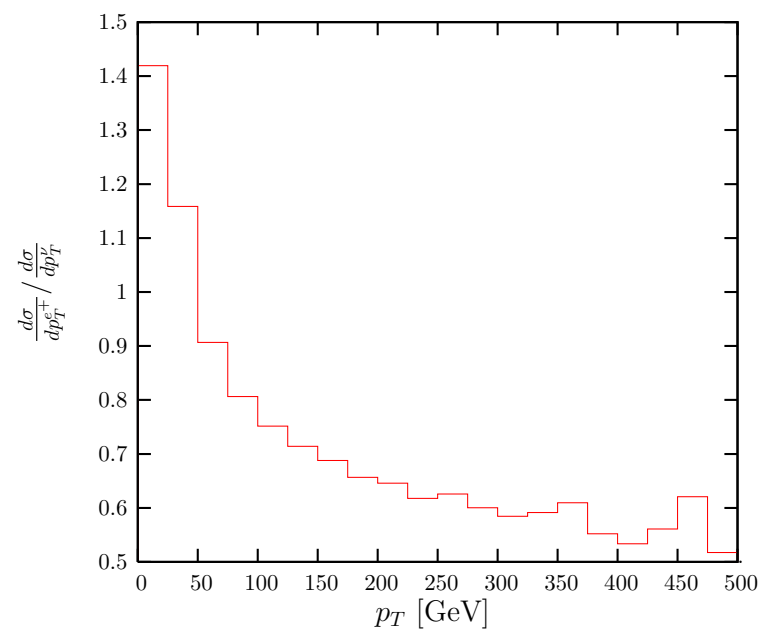

Figure 17: Ratio of differential cross sections for the charged lepton $p_{T}$ and the missing transverse energy for $W^{+}$production from top decay at the LHC. The plot is identical for $W^{-}$. A jet cut $p_{T}^{j}>30 \mathrm{GeV}$ has been imposed.

Higgs production and $H \rightarrow W^{+} W^{-}$decay. In this section we compare the polarisation properties of these $W$ bosons with those from standard QCD $W+$ jets production. In each case we use the same projections as for $W+$ jets defined in Section 2 and select the available MCFM subprocesses where the $W^{+}$decays to a positron and a neutrino and the other particles produced in the event $\left(W^{-}, H, Z\right)$ decay hadronically so that for each event there is only one charged lepton present.

We first note that the polarisation fractions of the produced $W^{+}$boson vary from process to process as shown in the plots of Fig. 20, for which no selection cuts have been applied. For each case we also give the LO positron channel subprocess cross section result obtained using MCFM as a guide to the relative importance of each process as a $W$ source. $^{3}$

\footnotetext{
${ }^{3}$ Similar calculations can of course be made for $W^{-}$production but here we only show the results for
} 


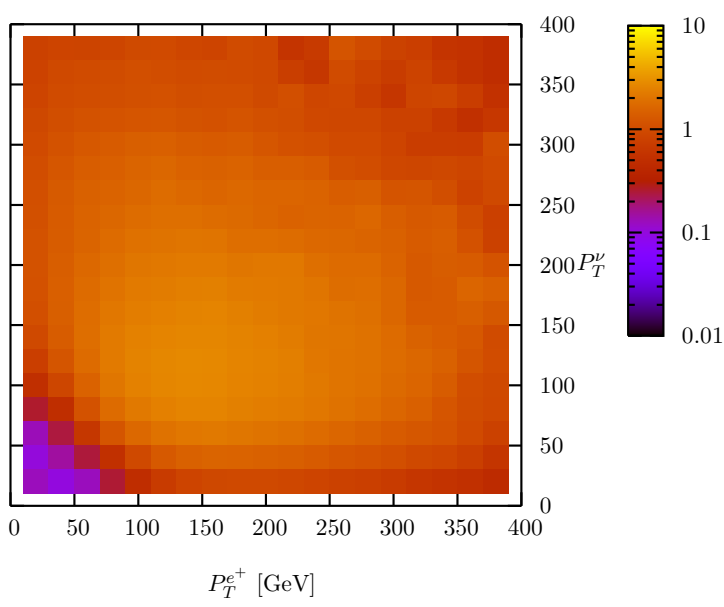

(a)

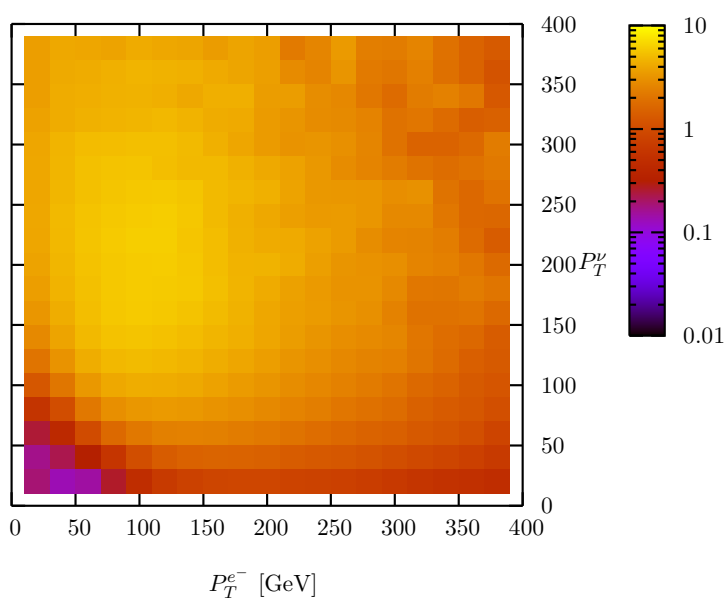

(b)

Figure 18: Density plots of the ratio of the double differential cross section for $t \bar{t}$ to $W+$ jets for a) $W^{+}$and b) $W^{-}$.

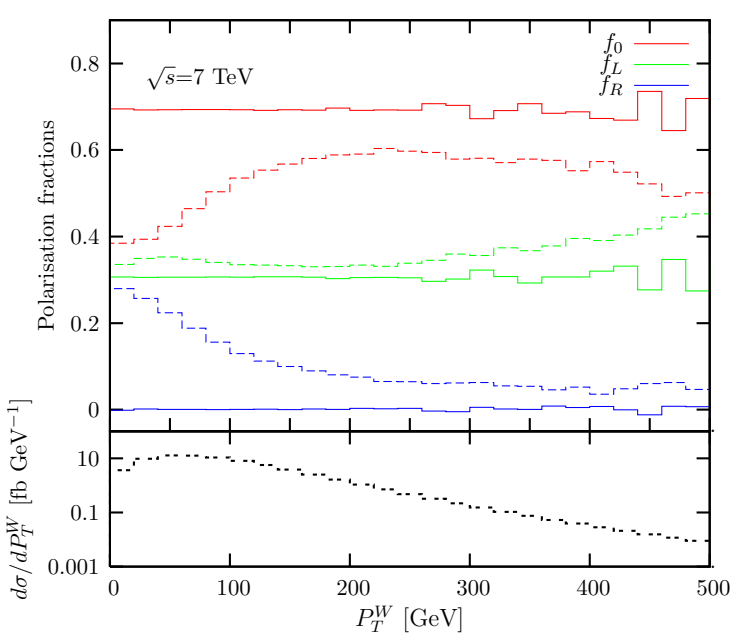

Figure 19: Polarisation fractions for $W^{+}$from top decays with no imposed cuts using the two angle definitions. Solid: top rest frame and dashed: lab frame.

These processes are in general a subdominant source of $W$ bosons compared to QCD $W+$ jets but it is still important to explore them both because they constitute a further set of backgrounds for New Physics searches and also because they are interesting processes in their own right. The cross-section results at $7 \mathrm{TeV}$ and the total polarisation fractions are given in Table 2. In all cases the $W^{+}$decays leptonically to a positron and a neutrino leading to missing transverse energy. In comparison with the distributions we note that the total fractions are more influenced by the fractions at low $p_{T}^{W}$ as in general the cross sections are rapidly decreasing functions at large $p_{T}^{W}$. The only cut imposed for the results shown in Table 2 is a jet $p_{T}$ cut of $30 \mathrm{GeV}$ for the $W+$ jets processes. As the cross

$W^{+}$ 

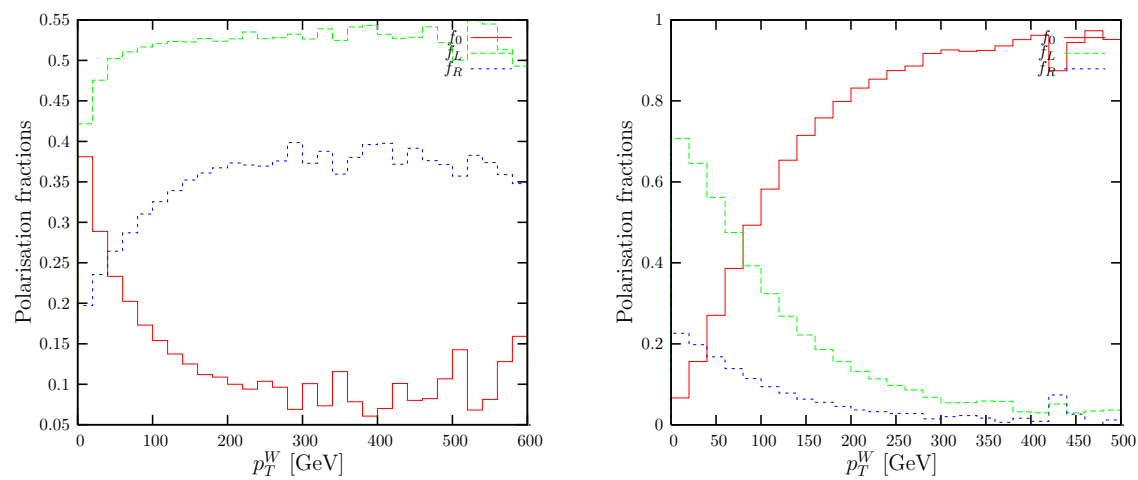

(a) $W$ pair production $\left(\mathrm{W}^{-}\right.$decays hadronically): $2048 \mathrm{fb}$

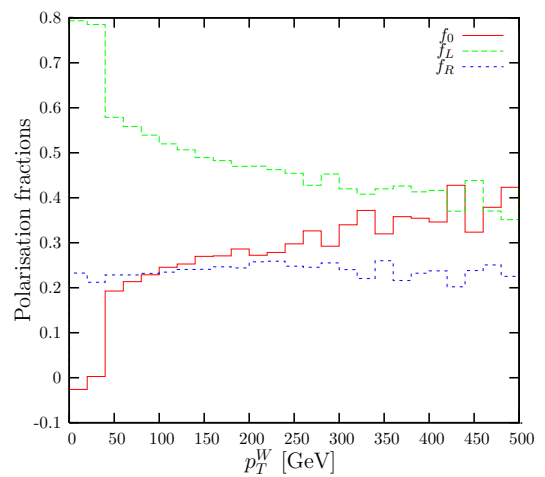

(b) $W+$ Higgs $\left(m_{H}=120 \mathrm{GeV}\right.$, Higgs decays to $b \bar{b}): 53 \mathrm{fb}$

(c) $W+Z(Z$ decays hadronically to $3 \times d \bar{d})$ : $622 \mathrm{fb}$
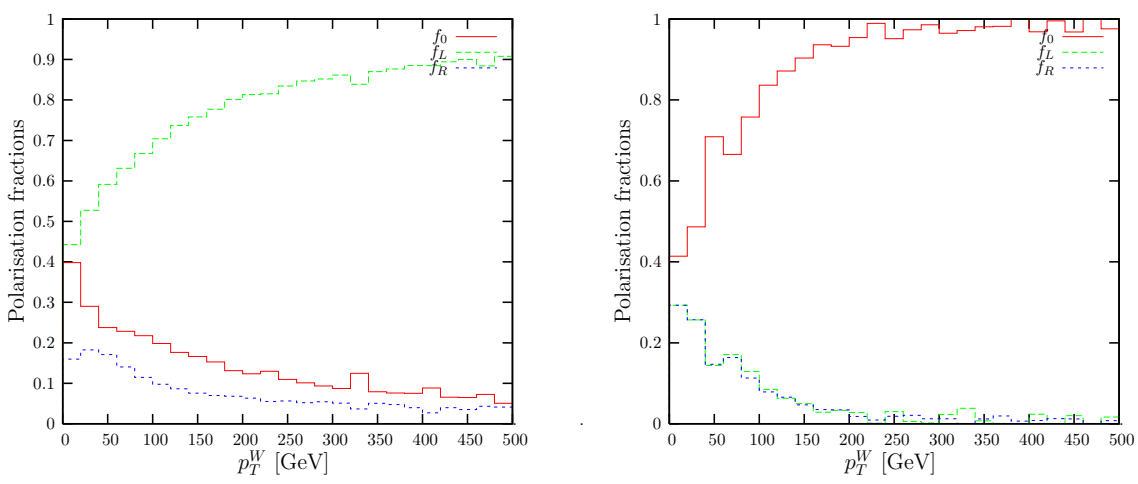

(e) Single top $s$-channel: $205 \mathrm{fb}$

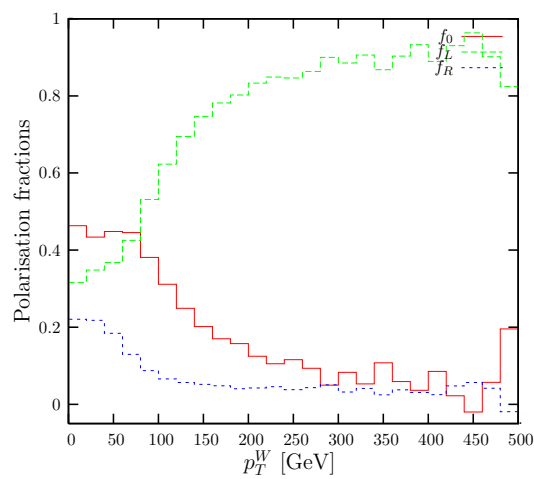

(d) Single top $t$-channel: $4067 \mathrm{fb}$

(f) $W$ pair from Higgs decay $\left(m_{H}=\right.$ $120 \mathrm{GeV}): 10.3 \mathrm{fb}$

Figure 20: Polarisation fractions for different processes.

sections for the non-QCD processes are much smaller, the experimental determination of the polarisation fractions could well be impeded by low statistics.

For single top production we also show, in Fig. 21, the result obtained using the projections defined in [17]. We note that the two definitions give results that coincide at high $p_{T}^{W}$, which is explained by the fact that the $W$ direction in the top rest frame and the lab frame coincide for large $p_{T}^{W}$. At very high $p_{T}^{W}$, the left-handed $b$-quark is preferentially 
Table 2: Comparison of results for the different processes leading to $W^{+}$production.

\begin{tabular}{|c|c|c|c|c|}
\hline Process & Cross section[fb] & $f_{0}$ & $f_{L}$ & $f_{R}$ \\
\hline$W+1$ jet $\left(p_{T}^{j}>30 \mathrm{GeV}\right)$ & $6.11 \cdot 10^{5}$ & 0.20 & 0.56 & 0.24 \\
\hline$W+2 \operatorname{jets}\left(p_{T}^{j}>30 \mathrm{GeV}\right)$ & $2.15 \cdot 10^{5}$ & 0.20 & 0.56 & 0.23 \\
\hline$W+3 \operatorname{jets}\left(p_{T}^{j}>30 \mathrm{GeV}\right)$ & $0.74 \cdot 10^{5}$ & 0.21 & 0.56 & 0.23 \\
\hline$t \bar{t}(\bar{t} \rightarrow b q \bar{q})$ & 1489 & 0.46 & 0.37 & 0.17 \\
\hline Single top $(t$-channel $)$ & 4067 & 0.42 & 0.43 & 0.15 \\
\hline Single top $(s$-channel $)$ & 205 & 0.24 & 0.61 & 0.14 \\
\hline$W+Z(Z \rightarrow 3 \times(d \bar{d}))$ & 622 & 0.05 & 0.72 & 0.23 \\
\hline$W+H(H \rightarrow b \bar{b})$ & 53 & 0.05 & 0.72 & 0.23 \\
\hline$W$ pair $\left(W^{-} \rightarrow q \bar{q}\right)$ & 2048 & 0.26 & 0.48 & 0.25 \\
\hline$W$ pair from $H\left(m_{H}=120 \mathrm{GeV}, H \rightarrow 4 l\right)$ & 10.3 & 0.46 & 0.27 & 0.27 \\
\hline
\end{tabular}

produced antiparallel to the top direction and the left-handed $W$ is produced parallel to the top by angular momentum conservation. The sum of the $W$ and $b$ momenta gives the top momentum and therefore the $W$ transverse momentum is on average larger than that of the top - the top 'sees' the $W$ boosted in the same direction as seen in the lab frame.

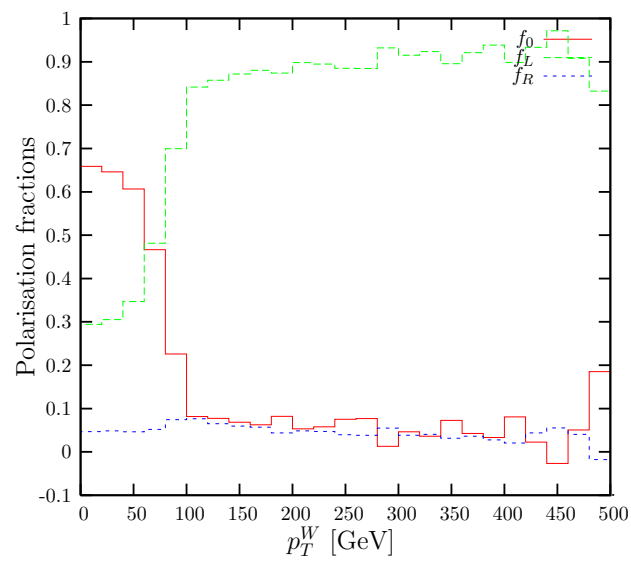

(a)

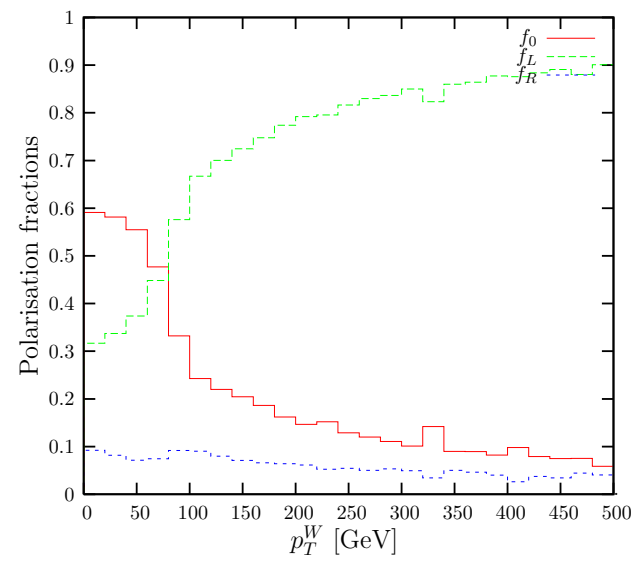

(b)

Figure 21: Polarisation fractions for $W^{+}$from single top a) $t$-channel and b) $s$-channel production using the direction of the $W$ in the top rest frame.

It is clear from the plots and from Table 2 that the polarisation properties of $W$ bosons depend strongly on the production process. One could try to explain the results based on the helicities of the particles involved at scattering amplitude level, as attempted in Ref. [5] for $\mathrm{QCD} W+1$ jet production. However this rapidly becomes complicated and no clear conclusions can be drawn.

One further step that could be taken is to try to locate the origin of the differences between a pair of processes. We expect the difference between two processes to be partly 
caused by different kinematics and partly by the physics of the underlying interactions. In order to disentangle the effect of kinematics from the interaction we can impose cuts on one process to mimick the kinematics of another process.

As an example we compare $W+Z$ associated production where the $Z$ decays hadronically with QCD $W+2$ jets production where we impose a cut on the jet pair invariant mass to force it to be very close to the $Z$ mass. The effect of this constraint is shown in Fig. 22. Of course we must bear in mind that in $W+Z$ production the $Z$ can be off-shell and also that a small admixture of photon events is included but we can check this by examining the invariant mass of the two final state jets for this process. In the dijet mass differential cross section we can see the rapid increase around the $Z$ mass pole. There is also an increase at small invariant masses due to the contribution of the virtual photon which is also included in the MCFM calculation. By selecting the appropriate subprocess in which the $Z$ decays to neutrinos and therefore no photon events can be included, we can examine the impact of the photon events in the polarisation properties of the $W$ boson. The shape is only modified at small transverse momentum, but the basic characteristics at high $p_{T}$ persist even though the crossing between $f_{L}$ and $f_{0}$ shifts to a higher $p_{T}$ value.

Another consideration in this comparison is the dominant parton subprocess for the two processes. For $W+2$ jets production at the LHC quark-gluon scattering dominates over quark-quark scattering. On the other hand at LO $W+Z$ production originates only from quark-antiquark scattering. To extract more information we can decompose the $W+2$ jets cross section into contributions from quark and gluon scattering components by setting the gluon PDF to zero. The results for this are shown in Fig. 23. As the imposed constraints

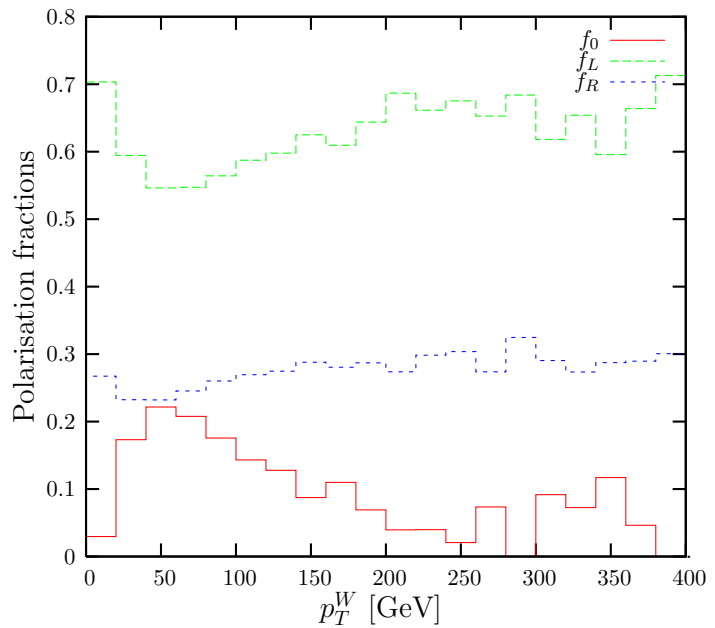

Figure 22: Polarisation fractions for $W^{+}+$ 2 jets production for the dijet mass of the jet pair constrained to be close to $M_{Z}$.

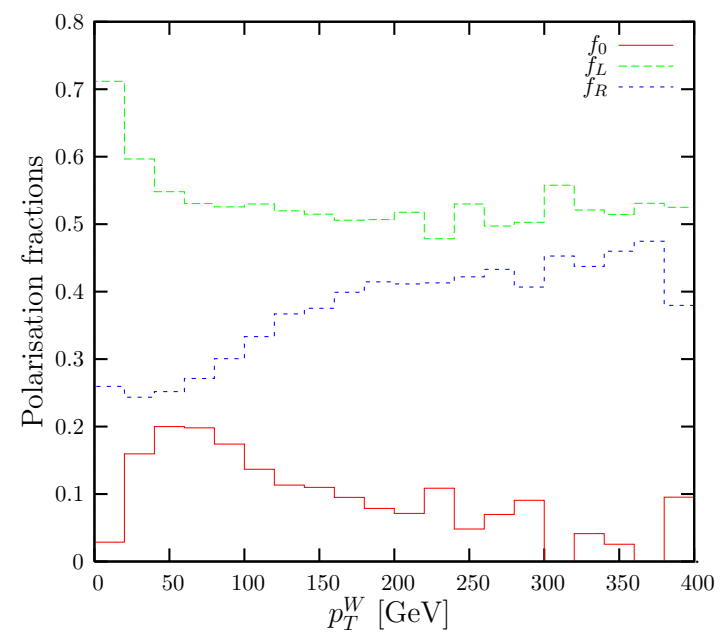

Figure 23: Polarisation fractions for quark only contribution to $W+2$ jets production mimicking the kinematics of $W+Z$.

decrease the cross section dramatically, lack of statistics inevitably affects the results at high $p_{T}$. Comparing this to the result for $W+Z$, we notice that the shapes remain quite different especially at high $p_{T}^{W}$. Therefore the polarisation behaviour here originates mainly from the underlying interaction. This is consistent with the fact that for $W+$ jets 
production with no initial state gluons the two outgoing jets are gluon jets of QCD origin. On the other hand, in the $W+Z$ case the two jets are quark jets that originate from an electroweak interaction that also distinguishes fermion helicities through the right-handed and left-handed couplings.

Also related to the underlying interaction in the process producing the $W$ boson is the similarity in the shapes of the results for $W+H$ associated production and $H \rightarrow W^{+} W^{-}$ Higgs decay. In both cases the $W$ boson is predominantly longitudinal at high $p_{T}$ which is related to the spin- 0 nature of the Higgs boson involved in the interaction. The difference between the two is the absence of an asymmetry between left and right polarisation for $W$ from Higgs decay. The asymmetry observed in $W H$ production at small $p_{T}$ is related to the initial state quark-anti-quark distributions producing a preferentially left-handed $W$.

As already stressed above, these are complicated processes and it is not straightforward to predict the results in a simple analysis such as that performed in [5] for $W+1$ jet production. One region for which we do have some intuition is the region of high $W p_{T}$ for $W+Z, W+H$ and single top production using a simple angular momentum conservation argument. For $W+H$ production, by considering that only left-handed quarks are involved and that the Higgs is a scalar, we expect the $W$ to be predominantly longitudinal. On the other hand, for $W+Z$ production we expect the $f_{L}$ and $f_{R}$ polarisation fractions to be the same. This can be seen by drawing angular momentum conservation diagrams with the momentum and spin vectors of each particle, similar to those used in [5] for $W+1$ jet production.

We also note that $f_{0} \rightarrow 0$ at large $p_{T}^{W}$ except for $W$ plus Higgs and $W+Z$. This is related to the equivalence theorem which states that at high energies the longitudinally polarised gauge boson states can be replaced by the corresponding Goldstone bosons which do not couple to light quarks. For the processes involving only light quarks this implies that $f_{0} \rightarrow 0$ at high energies.

\section{5. $Z$ boson polarisation}

The methods defined and employed for $W$ bosons can in principle also be used to measure the polarisation of $Z$ bosons produced at the LHC. In the case of the $Z$ boson decaying to two charged leptons, the shape of the positively and negatively charged lepton distributions cannot be used as a probe of polarisation in the same way as for the $W$ boson, because the $Z$ couples to both right- and left-handed fermions. Therefore the SM leptonic couplings need to be taken into account and the equivalent of Eq. (2.1) for $Z$ decay to a pair of fermions is:

$$
\begin{aligned}
\frac{1}{\sigma} \frac{d \sigma}{d \cos \theta^{*}} & =\frac{3}{8}\left(1+\cos ^{2} \theta^{*}-\frac{2\left(c_{L}^{2}-c_{R}^{2}\right)}{\left(c_{L}^{2}+c_{R}^{2}\right)} \cos \theta^{*}\right) f_{L}+\frac{3}{8}\left(1+\cos ^{2} \theta^{*}+\frac{2\left(c_{L}^{2}-c_{R}^{2}\right)}{\left(c_{L}^{2}-c_{R}^{2}\right)} \cos \theta^{*}\right) f_{R} \\
& +\frac{3}{4} \sin ^{2} \theta^{*} f_{0}
\end{aligned}
$$

with $c_{R}$ and $c_{L}$ the right- and left-handed couplings of the fermion to the $Z$ and $\theta^{*}$ the angle measured in the $Z$ rest frame between the antiparticle and the $Z$ flight direction 
in the lab frame. Based on Eq. (5.1) and similarly to $W$ boson production we can use appropriate projections, which are however in this case dependent on the couplings, to obtain the polarisation fractions:

$$
\begin{aligned}
& f_{0}=2-5\left\langle\cos ^{2} \theta^{*}\right\rangle \\
& f_{L}=-\frac{1}{2}-\frac{\left(c_{L}^{2}-c_{R}^{2}\right)}{\left(c_{L}^{2}+c_{R}^{2}\right)}\left\langle\cos \theta^{*}\right\rangle+\frac{5}{2}\left\langle\cos ^{2} \theta^{*}\right\rangle, \\
& f_{R}=-\frac{1}{2}+\frac{\left(c_{L}^{2}-c_{R}^{2}\right)}{\left(c_{L}^{2}+c_{R}^{2}\right)}\left\langle\cos \theta^{*}\right\rangle+\frac{5}{2}\left\langle\cos ^{2} \theta^{*}\right\rangle .
\end{aligned}
$$

For the decay to neutrinos the projections are identical to those for $W$ bosons, since $c_{R}^{\nu}=0$. Using our MC generators we can use different decay channels to determine the polarisation fractions of $Z$ bosons. In contrast to the case of $W$ bosons, for which $\theta^{*}$ cannot be extracted precisely due to the unreconstructed longitudinal $W$ momentum, for $Z$ production $\theta^{*}$ is unambiguously defined and reconstructed. Therefore there is no need to use the variable $L_{p}$ introduced earlier. This method can therefore be used directly to determine the polarisation of $Z$ bosons from different processes. Here we briefly comment on QCD $Z+$ jets production and $Z$ bosons produced in association with $W$.

\section{$5.1 Z$ plus jets}

The polarisation results are shown in Fig. 24 for $Z+1$ jet at $7 \mathrm{TeV}$ with a jet $p_{T}$ cut of $30 \mathrm{GeV}$. Evidently $Z$ bosons are also predominantly left-handed at non-zero transverse momentum. Comparing the results for $Z$ to those for $W+1$ jet we note that while the $Z$ is also predominantly left-handed, the exact values of the fractions differ, as they arise from a combination of the different quark flavour combinations producing the $Z$ boson and the fact that the $Z$ couples to both left- and right-handed quarks. A complication that arises in $Z$ boson studies is the small admixture of photon events. In terms of the polarisation fraction expressions given above, these apply only to pure $Z$ exchange. Experimentally this is limited by introducing a cut constraining the lepton pair invariant mass to be close to the $Z$ mass. In practice, for example in the CMS $Z$ boson analysis [30], this is constrained to lie between 60 and $120 \mathrm{GeV}$. Using MCFM we can allow for the inclusion of photon events in this region of masses, obtaining the $\mathrm{LO}$ cross section for $Z+1$ jet production with a jet $p_{T}$ cut of $30 \mathrm{GeV}$ of $105.9 \mathrm{pb}$ when these are included and $103.3 \mathrm{pb}$ when they are excluded. Given the small percentage difference and the appropriate use of MC generators a determination of the polarisation fractions of $Z$ from the data sample should therefore be possible.

\section{$5.2 W$ plus $Z$}

Similarly to the measurement of the polarisation of the $W$ in $W Z$ production, we can extract the polarisation of the $Z$ in the same process. For this we use the MCFM subprocess for which the $Z$ decays to neutrinos to avoid the small admixture of photon events. The results are shown in Fig. 25 as a function of the $Z$ transverse momentum and the centre-ofmass energy. We note the increase in $f_{0}$ at high $p_{T}$. This can also be explored by considering 


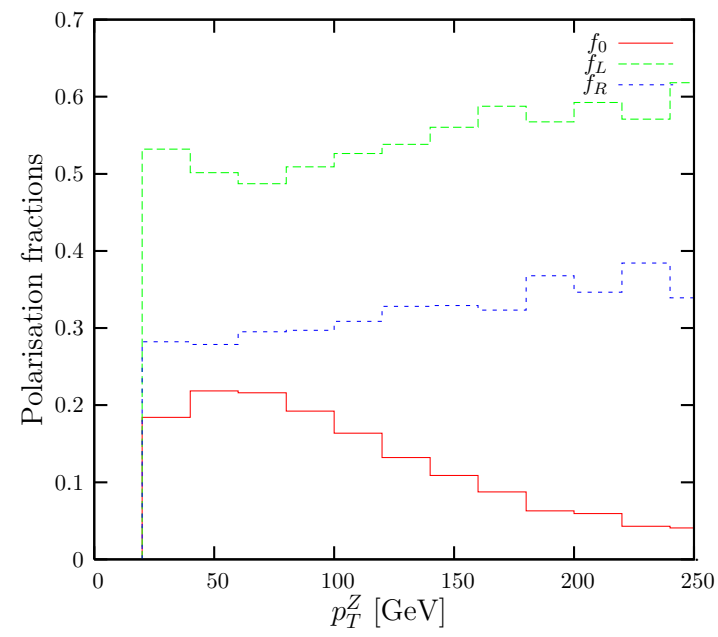

Figure 24: $Z$ polarisation fractions from the electron decay channel.

the polarisation fractions as a function of the centre-of-mass energy from which we see that $f_{0}$ falls to zero at high energies. One might expect that high $p_{T}$ corresponds to high centreof-mass energy and therefore that the limiting values should coincide. The fact that this is not observed to be the case can be investigated by setting a cut on the centre-of-mass energy and computing the $p_{T}$ distribution, and vice versa. Setting a cut $E_{c}$ on the centre-of-mass energy results in modified polarisation fractions at low $p_{T}$ while the fractions remain the same for $p_{T}>E_{c} / 2$. In contrast, imposing a cut $E_{c} / 2$ on the $p_{T}$ forces the centre-of-mass energy to be larger than $E_{c}$ but also modifies the polarisation fractions at any given energy above $E_{c}$. These observations for the centre-of-mass energy and transverse momentum can explain the different shapes of the polarisation fraction distributions and the more complex connection between Figs. 25 a) and b). Considering the differential distributions for $p_{T}^{Z}$ and centre-of-mass energy also helps us verify that even though the shapes are different they result as expected in the same total polarisation fractions when integrated over the whole phase space. Moreover we have checked that, as expected, when processes allowing photons are selected, at very low lepton pair invariant masses where photon events dominate, it can be seen that $f_{0}$ vanishes, as photons can only be transversely polarised.

Another observation that can be made by computing the polarisation fractions is the different behaviour of the polarisation of $Z$ in $W^{+}+Z$ compared to $W^{-}+Z$. This is not unexpected, as in one case the $Z$ is produced from a $u \bar{u}$ pair and in the other from a $d \bar{d}$ pair. The different right- and left-handed couplings to these fermions then leads to different polarisation fractions.

\section{Conclusions}

We have studied the angular distributions of $W$ boson decay products to extract information on the corresponding polarisation fractions. We have seen that $W$ bosons produced in association with QCD jets at non-zero transverse momentum are preferentially left-landed at the LHC. This leads to asymmetries between the charged lepton and neutrino transverse 


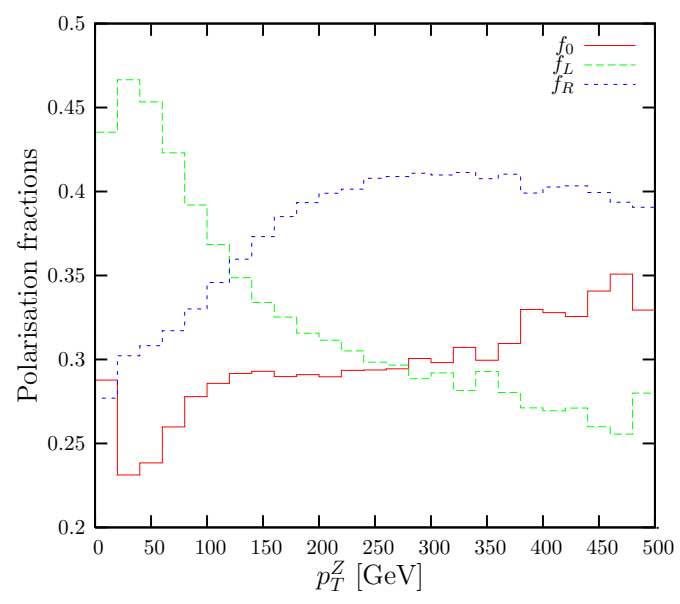

(a)

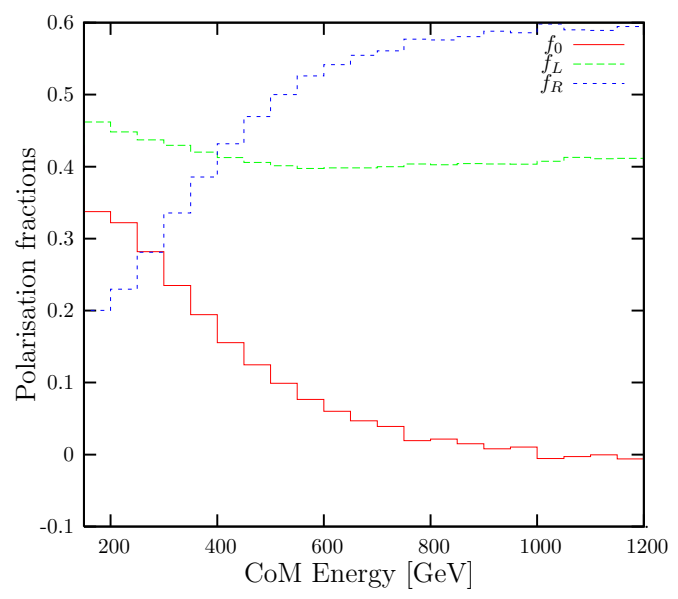

(b)

Figure 25: Polarisation fractions for $Z$ bosons from $W^{+}+Z$ production as a function of a) the $Z p_{T}$ and b) the centre-of-mass energy.

momentum distributions. The dependence of the angular distributions has been studied for different selection cuts and these were found to change rapidly on the introduction of cuts and therefore experimentally it will be necessary to correct for the cuts before extracting polarisation fraction information.

We have compared the polarisation of $W$ bosons produced with QCD jets to $W$ bosons from top pair production and decay, calculating the polarisation fractions in the same frame and comparing the shape of different observable distributions and the asymmetry between the lepton transverse momentum and the missing transverse energy. We have also compared the polarisation fractions obtained in two different frames, to show that the polarisation fractions are strongly frame dependent. We remark that the $f_{0}=0.7, f_{L}=0.3$ fractions often mentioned in the literature are valid only when defining the relevant angle in the top rest frame.

We have used the same procedure for other $W$ producing processes at the LHC to study the polarisation of $W$ bosons as a function of the $W$ transverse momentum. Other processes have lower cross sections and measurements of the polarisation properties could well be impeded by low statistics, at least at present. However with increasing LHC luminosity, it should in the near future become possible to extract the $W$ polarisation fractions in the same way as has been done by the CMS and ATLAS collaborations for $W+$ jets production. Similarly, we expect the measurement of the $Z$ polarisation to be feasible at the LHC and have presented the relevant results.

Comparing different $W$ processes with very different polarisation results we note that the origin of the difference is related to the underlying physics of the interaction and the helicities of the other particles involved. Therefore a study of the polarisation properties can be used in conjunction with kinematics to distinguish between different sources of $W$ bosons. This is also helpful for New Physics searches where new interactions might give different polarisation fractions and can therefore be used as a handle to disentangle the 
signal from the SM background.

\section{Acknowledgments}

E.V. acknowledges financial support from the UK Science and Technology Facilities Council. We thank Lance Dixon and Marco Peruzzi for useful discussions.

\section{References}

[1] C. F. Berger, Z. Bern, L. J. Dixon, F. Febres Cordero, D. Forde, T. Gleisberg, H. Ita and D. A. Kosower et al., Phys. Rev. Lett. 106, 092001 (2011) [arXiv:1009.2338 [hep-ph]].

[2] H. Ita, Z. Bern, L. J. Dixon, F. F. Cordero, D. A. Kosower and D. Maitre, arXiv:1108.2229 [hep-ph].

[3] R. K. Ellis, W. J. Stirling and B. R. Webber, Camb. Monogr. Part. Phys. Nucl. Phys. Cosmol. 8, 1 (1996).

[4] C. F. Berger, Z. Bern, L. J. Dixon, F. Febres Cordero, D. Forde, T. Gleisberg, H. Ita and D. A. Kosower et al., Colliders," Phys. Rev. D 80, 074036 (2009) [arXiv:0907.1984 [hep-ph]].

[5] Z. Bern, G. Diana, L. J. Dixon, F. Febres Cordero, D. Forde, T. Gleisberg, S. Hoeche and H. Ita et al., Phys. Rev. D 84, 034008 (2011) [arXiv:1103.5445 [hep-ph]].

[6] S. Chatrchyan et al. [CMS Collaboration], Phys. Rev. Lett. 107, 021802 (2011) [arXiv:1104.3829 [hep-ex]].

[7] G. Aad et al. [ATLAS Collaboration], arXiv:1203.2165 [hep-ex].

[8] D. Acosta et al. [CDF Collaboration], Phys. Rev. D 73, 052002 (2006) [hep-ex/0504020].

[9] J. C. Collins and D. E. Soper, Phys. Rev. D 16, 2219 (1977).

[10] C. S. Lam and W. -K. Tung, Phys. Rev. D 21, 2712 (1980).

[11] E. Mirkes, Nucl. Phys. B 387, 3 (1992).

[12] E. Mirkes and J. Ohnemus, Phys. Rev. D 50, 5692 (1994) [hep-ph/9406381].

[13] V. M. Abazov et al. [D0 Collaboration], Phys. Rev. Lett. 100, 062004 (2008) [arXiv:0711.0032 [hep-ex]].

[14] V. M. Abazov et al. [D0 Collaboration], Phys. Rev. D 83, 032009 (2011) [arXiv:1011.6549 [hep-ex]].

[15] T. Aaltonen et al. [CDF Collaboration], Phys. Lett. B 674, 160 (2009) [arXiv:0811.0344 [hep-ex]].

[16] T. Aaltonen et al. [The CDF Collaboration], Phys. Rev. Lett. 105, 042002 (2010) [arXiv:1003.0224 [hep-ex]].

[17] J. A. Aguilar-Saavedra, J. Carvalho, N. F. Castro, F. Veloso and A. Onofre, Eur. Phys. J. C 50, 519 (2007) [hep-ph/0605190].

[18] C. L. Bilchak, R. W. Brown and J. D. Stroughair, Phys. Rev. D 29, 375 (1984).

[19] M. J. Duncan, G. L. Kane and W. W. Repko, Nucl. Phys. B 272, 517 (1986).

[20] S. S. D. Willenbrock, Annals Phys. 186, 15 (1988). 
[21] P. Achard et al. [L3 Collaboration], Phys. Lett. B 557, 147 (2003) [hep-ex/0301027].

[22] G. Abbiendi et al. [OPAL Collaboration], Phys. Lett. B 585, 223 (2004) [hep-ex/0312047].

[23] J. Abdallah et al. [DELPHI Collaboration], Eur. Phys. J. C 54, 345 (2008) [arXiv:0801.1235 [hep-ex]].

[24] A. D. Martin, W. J. Stirling, R. S. Thorne and G. Watt, Eur. Phys. J. C 63, 189 (2009) [arXiv:0901.0002 [hep-ph]].

[25] C. -H. Kom and W. J. Stirling, Eur. Phys. J. C 69, 67 (2010) [arXiv:1004.3404 [hep-ph]].

[26] J. M. Cornwall, D. N. Levin and G. Tiktopoulos, Phys. Rev. D 10, 1145 (1974) [Erratum-ibid. D 11, 972 (1975)].

[27] J. M. Campbell and R. K. Ellis, http://mcfm.fnal.gov/.

[28] T. Gleisberg, S. Hoeche, F. Krauss, A. Schalicke, S. Schumann and J. -C. Winter, JHEP 0402, 056 (2004) [hep-ph/0311263].

[29] Z. Bern, G. Diana, L. J. Dixon, F. Febres Cordero, D. Forde, T. Gleisberg, S. Hoeche and H. Ita et al., arXiv:1201.5288 [hep-ph].

[30] S. Chatrchyan et al. [CMS Collaboration], arXiv:1110.4973 [hep-ex]. 